\title{
Model Predictive Control of Modular Multilevel Converters using Quadratic Programming
}

\author{
Jiapeng Yin, Student Member, IEEE, Jose I. Leon, Fellow, IEEE, Marcelo A. Perez, Senior Member, IEEE, \\ Leopoldo G. Franquelo, Life Fellow, IEEE, Abraham Marquez, Member, IEEE, \\ and Sergio Vazquez, Senior Member, IEEE
}

\begin{abstract}
The finite control set-model predictive control (FCSMPC) has been adopted as an excellent choice for the applications of multilevel converters during the last two decades for its salient performance. However, in the case of modular multilevel converters (MMCs), a high amount of calculation is always involved in the implementation, making the FCS-MPC less suitable especially for an MMC with high number of submodules (SMs). To cope with the issue, this paper proposes an MPC technique for the MMC with very low calculation cost. In each sampling period, the arm voltage references of each phase are determined analytically by solving a constrained quadratic programming problem formulated from the cost function. Both a rigorous and a simplified procedure are provided to solve the optimization problem. Then, the four nearest candidates around the arm voltage references are evaluated, leading to a proper selection of arm voltage levels. Several experimental tests on an MMC prototype are carried out to validate the effectiveness of the proposed method. Results show that compared with the conventional FCS-MPC method which evaluates all voltage-level combinations, the proposed scheme presents apparent advantage in terms of calculation cost while achieving similar performance.
\end{abstract}

Index Terms-Model predictive control (MPC), modular multilevel converters, quadratic programming.

\section{NOMENCLATURE}

$\begin{array}{ll}N & \text { Number of SMs per arm of the MMC. } \\ x & \in\{a, b, c\} . \text { Phase identifier. } \\ y & \in\{u, l\} \text { (upper, lower). Arm identifier. } \\ z & \in\{1, \ldots, N\} . \text { SM identifier. } \\ L_{\text {arm }} & \text { Inductance of arm inductor. }\end{array}$

Manuscript received June 8th, 2020; revised August 31st, 2020; accepted October 22nd, 2020. This work was supported in part by the Spanish Science and Innovation Ministry under project TEC2016-78430-R and Consejeria de Economia, Conocimiento, Empresas y Universidad, in part by the Secretaria General de Universidades, Investigacion y Tecnologia under the project PY181340 , in part by the 2020 horizon SPARTAN project (ID 821381), in part by the Fondecyt Project 1181839, in part by the Advanced Center for Electrical and Electronics Engineering AC3E (ANID/FB0008), and in part by the Chilean Solar Energy Research Center (ANID/FONDAP/15110019). (Corresponding author: Jiapeng Yin.)

J. Yin, A. Marquez and S. Vazquez are with the Department of Electronic Engineering, Universidad de Sevilla, 41092 Seville, Spain (e-mail: jpyin1992@gmail.com; amarquez@iee.org; sergi@us.es).

J. I. Leon and L. G. Franquelo are with the Laboratory of Engineering for Energy and Environmental Sustainability, Department of Electronic Engineering, Universidad de Sevilla, 41092 Seville, Spain, and also with the Department of Control Science and Engineering, School of Astronautics, Harbin Institute of Technology, Harbin 150001, China (e-mail: jileon@gte.esi.us.es; lgfranquelo@ieee.org).

M. A. Perez is with the Department of Electronics, Universidad Técnica Federico Santa María, Valparaiso 2390123, Chile (e-mail: marcelo.perez@usm.cl).

$R_{a r m}$
$C$
$V_{d c}$
$i_{d c}$
$v_{S x}$
$L$
$R$
$R$
$v_{C x y z}$
$v_{x y}$
$v_{d c x y}$
$i_{x y}$
$i_{x}$
$i_{c i r c, x}$
$W_{x y}$
$W_{x \Delta}$
$W_{x \Sigma}$
$N_{x y}$
$S_{x y z}$
$f$
$T_{s}$
$k$
$J_{i}$
$J_{w}$
$J_{i w}$

$p_{1,2,3,4}$

$\lambda_{1,2,3,4}$

$f_{1,2,3,4}, g_{1,2}$

$v_{x y}$

$v_{x y \_0}$

$v_{x y_{-} 0}$

$Q_{i}, c_{i}$

$Q_{w}, c_{w}$

$Q_{i w}, c_{i w}$
Parasitic resistance of arm.

Capacitance of SM floating capacitor.

DC-link voltage.

DC-link current.

Grid voltage of phase- $x$.

Grid-side inductance (or load inductance in case of load connection).

Grid-side resistance (or load resistance in case of load connection).

Capacitor voltage of SM-xyz.

Arm voltage of arm- $x y$.

Sum of SM capacitor voltages of arm- $x y$.

Arm current of arm- $x y$.

Phase current of phase- $x$.

Circulating current of phase- $x$.

Arm energy of all SMs of arm-xy.

Difference of both arm energies of phase- $x$.

Sum of both arm energies of phase- $x$.

Number of switched on SMs (insertion index) of arm- $x y$.

Switching state of SM-xyz.

AC side frequency.

Sampling/control period.

Integer representing a certain time step in the discrete time domain.

Cost function considering current tracking.

Cost function considering arm-energy regulating.

Cost function considering both current tracking and arm-energy regulating.

Weighting factors of the original cost functions.

Weighting factors of the reformed cost functions.

Intermediate variables for cost-function reformulation.

Arm-voltage vector of phase- $x$.

Arm-voltage vector of phase- $x$ corresponding to the minimum value of the unconstrained cost function.

Element of $\boldsymbol{v}_{\boldsymbol{x} \boldsymbol{y}_{-} \mathbf{0}}$.

Real-valued coefficient matrices of quadratic function derived from $J_{i}$.

Real-valued coefficient matrices of quadratic function derived from $J_{w}$.

Real-valued coefficient matrices of quadratic 


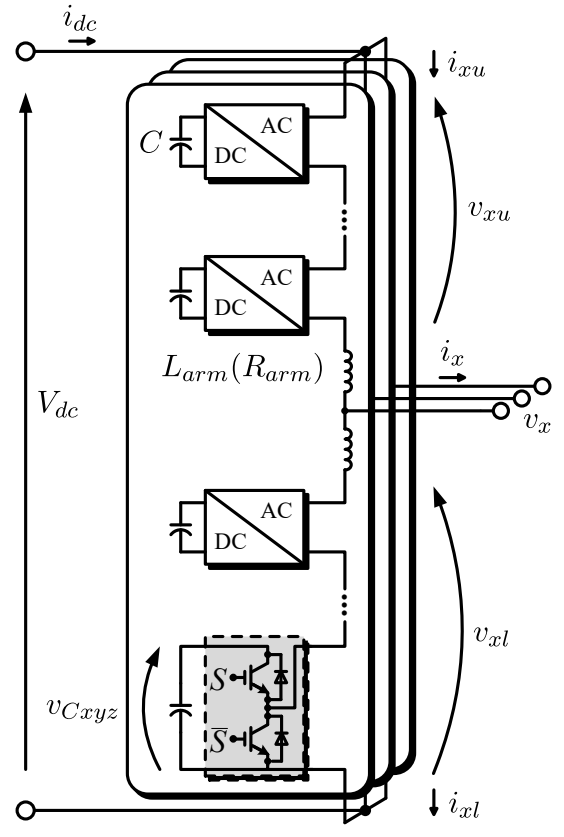

Fig. 1. Circuit configuration of the MMC.

$\begin{array}{ll} & \text { function derived from } J_{i w} . \\ q_{i 1,2,3}, c_{i 1,2} & \begin{array}{l}\text { Elements of } \boldsymbol{Q}_{\boldsymbol{i}} \text { and } \boldsymbol{c}_{\boldsymbol{i}} \text {, respectively. } \\ \boldsymbol{A}, \boldsymbol{b}\end{array} \\ & \begin{array}{l}\text { Real-valued coefficient matrices of constraint } \\ \text { of optimizing problems. }\end{array} \\ \mathrm{E}_{1,2} & \begin{array}{l}\text { Important tangent points in orthogonal coor- } \\ \text { dinate }\left(v_{x u}, v_{x l}\right) \text { for solving the optimization } \\ \text { problems. }\end{array} \\ & \text { Coordinate of } \mathrm{E}_{1,2} .\end{array}$

\section{INTRODUCTION}

T HE modular multilevel converter (MMC) has attracted great interest from power electronics academia and industry in recent years since 2003, when its concept was first proposed [1]. The structure of a three-phase MMC is illustrated in Fig. 1. Each phase leg consists of two arms and each arm is composed of a fixed number (denoted by $N$ ) of identical submodules (SMs) connected in series and a buffer inductor. The SM usually adopts the half-bridge topology as shown in Fig. 1. The modular structure of the MMC makes its output voltage and power highly scalable. Low voltage rating and low switching frequency of individual power devices and high-quality waveforms are also among the benefits from its topology. Owing to these features, the MMC has been extensively employed in high voltage/power applications [2][6].

However, the unique structure of the MMC also presents several challenges. To begin with, the SM capacitor voltages have unavoidable ripples, which then give rise to the circulating currents. Both detrimental effects lower the efficiency and power quality of the MMC system, even posing threat to the stability of the system if left uncontrolled. Therefore, effective modulation and control techniques are essential for the MMC operation in order to achieve high-quality output current (and therefore active/reactive power), i.e., the SMs need to be switched properly according to the operating conditions.

Main control objectives of an MMC generally include the regulation of phase currents, circulating currents and SMs capacitor voltages. As primary control objective, the phasecurrent control can be carried out in $a b c, \alpha \beta$ or $d q$ frame, aiming at a well tracking of the corresponding references given according to specific applications [3]. The circulatingcurrent controller, normally based on resonant controllers, is designed in most cases to eliminate the $\mathrm{AC}$ component for loss reduction, or to track DC-plus-AC references for SMcapacitor-voltage regulation [2]. The SM-capacitor voltages are normally controlled in two stages, namely the arm-totalcapacitor-voltage control (or arm-energy control) which regulates the DC component of SM capacitor voltages to the nominal value [7], and the capacitor-voltage balancing among the SMs within the same arm which is achieved depending on which modulation scheme is applied once the arm-voltage references are determined by the above-mentioned controllers. An extra individual capacitor-voltage-balancing controller is required for each SM if the gate signals are determined directly by the conventional pulse-width modulation (PWM) strategy [8], [9]. However, in high-power applications, the MMC usually contains several dozens or even hundreds of SMs per arm, thus making it complex to equip every SM with a carrier and an individual capacitor-voltage controller. From this perspective, it is preferable to determine the gate signals indirectly employing pseudomodulation techniques [10]: the number of SMs to be switched on (also called insertion index) of each arm is obtained first, followed by the sortingbased SM selection technique seeking for the balance of SM capacitor voltages. Among this stream of methods [7], [11][19], the nearest level control (NLC) [12], [13] is extensively adopted for its simple implementation, which is especially advantageous for high-power applications such as high-voltage direct current (HVDC) transmission [20].

Several finite control set-model predictive control (FCSMPC) schemes belong to this class [14], [15], where the insertion indexes of arms are determined employing predictive techniques, avoiding the huge amount of computation effort brought by evaluating all combinations of individual SM switching states. Normally, for each phase, different combinations of insertion indexes of the upper and lower arms are compared in order to select the optimal one, leading to $(N+1)^{2}$ candidates to be evaluated [15]. Several research works have dedicated effort to refine the candidates according to the operation [16]-[19]. However, the resulting size of control set is still, at least, proportional to $N$, and moreover, with possibility of losing optimality.

As an alternative of the FCS-MPC method, the continuous control set-MPC (CCS-MPC) scheme derives the output voltage reference analytically and then applies the modulation stage to generate the switching signals [21], [22]. In the context of the MMC, several CCS-MPC techniques have been derived during the last years [23]-[25], which normally integrate the PWM scheme to generate the derived arm voltage reference, leading to a better regulated harmonic spectrum.

In this paper, the FCS-MPC and the CCS-MPC techniques 
are both considered to develop a joint control method to operate the MMC. The core step is to determine the arm voltage references of the two arms of each phase according to the cost function which includes various control objectives of the system in terms of the regulation of output current, circulating current, and arm energy. In order to achieve this task, a quadratic programming problem is structured considering the range of each arm voltage as a constraint. After obtaining the arm voltage references, the variable rounding level control (VRLC) method developed in [26] is employed to select the optimal insertion indexes of both arms of each phase among four candidates determined by the arm voltage references.

The main contributions of this paper can be summarized as follows:

- The arm-voltage references which correspond to the minimum cost-function value under the specified feasible set are directly obtained employing quadratic programming technique, instead of evaluating many combinations of arm-voltage levels as required by conventional FCSMPC schemes, leading to optimal performance defined by the cost function in terms of phase-current tracking, circulating-current tracking and arm-energy regulation.

- Desired arm-voltage levels can be obtained with much reduced computational burden since only 4 evaluations of arm-voltage-level combinations per phase per control period are required, regardless of the number of SMs of the MMC, leading to similar (almost identical) performance with the conventional FCS-MPC method [15] that evaluates all the $(N+1)^{2}$ arm-voltage-level combinations.

- Both rigorous and simplified procedures are provided for the generation of arm-voltage references, which can be integrated into different MMC-based applications in various operating conditions by simply implementing the proposed method as the inner control loop and applying the conventional outer control loop for the determination of phase/circulating-current references.

- Further removing the barriers for the prospective industrial applications of the MPC techniques to the MMCs, especially those with large number of SMs in high voltage applications, allowing for an implementation based on microprocessors with reduced processing capability, leading to potential economic gains.

The rest of the paper is arranged as follows. Section II derives the constrained optimization problems from the system model and the cost function. The solutions are provided in section III. Section IV addresses the determination of arm voltage levels and gate signals. Then, section $\mathrm{V}$ and VI provide the experimental and simulation validation with comparative analysis based on the results. At last, section VII concludes the paper.

\section{FORMULATION OF OPTIMIZATION PROBLEM}

\section{A. System Model}

From the MMC topology shown in Fig. 1, the mathematical models of the output current and circulating current (of phase $x=a, b, c)$ can be obtained as

$$
\left(L_{a r m}+2 L\right) \frac{d i_{x}}{d t}=\left(-R_{a r m}-2 R\right) i_{x}-2 v_{S x}-v_{x u}+v_{x l}
$$
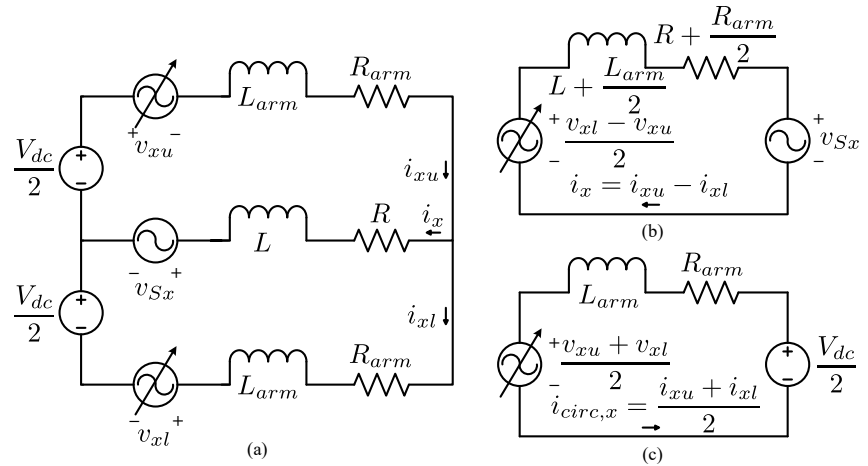

Fig. 2. Per-phase models of a grid-connected MMC system: (a) circuit of phase- $x$. (b) equivalent phase-current model. (c) equivalent circulating-current model.

$$
2 L_{a r m} \frac{d i_{c i r c, x}}{d t}=-2 R_{a r m} i_{c i r c, x}+V_{d c}-v_{x u}-v_{x l} .
$$

The $\mathrm{AC}$ side of the $\mathrm{MMC}$ is connected through an $R L$ filter to a grid with phase voltage of $v_{S x}$ and the common-mode voltage of its neutral point relative to that of the DC side is neglected since it is reasonable in a balanced operation. The model is flexible because in case of off-grid connection, $v_{S x}$ is equal to zero and $R, L$ denote the corresponding load parameters. To aid comprehension, the per-phase circuit of the investigated MMC-based system and the corresponding decoupled phase-current and circulating-current models are provided in Fig. 2.

The total energy of all SMs of the upper/lower arm is denoted by $W_{x y}(y=u, l)$. In the same phase $x$, the difference and sum of the total energy of both arms are defined as

$$
\begin{aligned}
& W_{x \Delta}=W_{x u}-W_{x l} \\
& W_{x \Sigma}=W_{x u}+W_{x l}
\end{aligned}
$$

respectively. Their dynamics are

$$
\begin{aligned}
& \frac{d W_{x \Delta}}{d t}=i_{x u} v_{x u}-i_{x l} v_{x l} \\
& \frac{d W_{x \Sigma}}{d t}=i_{x u} v_{x u}+i_{x l} v_{x l} .
\end{aligned}
$$

Applying the forward Euler approximation to (1), (2), (5) and (6) during time step $k$ to $k+1$ (with sampling time of $T_{s}$ ), the corresponding discrete models can be derived as

$$
\begin{array}{r}
\hat{i}_{x}(k+1)=i_{x}(k)+\frac{T_{s}}{L_{a r m}+2 L}\left[\left(-R_{a r m}-2 R\right) i_{x}(k)\right. \\
\left.-2 v_{S x}(k)-v_{x u}(k)+v_{x l}(k)\right]
\end{array}
$$

$$
\begin{array}{r}
\hat{i}_{c i r c, x}(k+1)=i_{c i r c, x}(k)+\frac{T_{s}}{2 L_{a r m}}\left[-2 R_{a r m} i_{c i r c, x}(k)\right. \\
\left.+V_{d c}-v_{x u}(k)-v_{x l}(k)\right]
\end{array}
$$

$$
\hat{W}_{x \Delta}(k+1)=W_{x \Delta}(k)+T_{s}\left[i_{x u}(k) v_{x u}(k)-i_{x l}(k) v_{x l}(k)\right]
$$

$$
\hat{W}_{x \Sigma}(k+1)=W_{x \Sigma}(k)+T_{s}\left[i_{x u}(k) v_{x u}(k)+i_{x l}(k) v_{x l}(k)\right]
$$


where the total arm energy is given as

$$
W_{x y}(k)=\frac{C}{2} \sum_{z=1}^{N} v_{C x y z}(k)^{2} \approx \frac{C v_{d c x y}(k)^{2}}{2 N}
$$

neglecting the difference between different SM capacitor voltages of the same arm. $v_{d c x y}$ is defined as the sum of all the SM capacitor voltages of the corresponding arm, also serving as the maximum of the arm voltage as

$$
0 \leq v_{x y}(k) \leq v_{d c x y}(k)=\sum_{z=1}^{N} v_{C x y z}(k) .
$$

\section{B. Cost Function}

1) Considering only current tracking: In order to employ the MPC technique, the cost function is introduced as

$$
J_{i}=p_{1}\left(i_{x}^{*}-\hat{i}_{x}\right)^{2}+p_{2}\left(i_{c i r c, x}^{*}-\hat{i}_{c i r c, x}\right)^{2}
$$

taking into account the output current and circulating current which are the most important control objectives of an MMC. $p_{1,2}>0$ denote the weighting factors and the variables with $*$ denote the corresponding references. The current references are determined by the outer control loop according to the applications and/or operating conditions (in most of the cases implemented by the conventional field-oriented control (FOC) and voltage-oriented control (VOC) for motor drives and grid connected applications respectively), which are considered here as given information and the investigation will be focused on the inner current control loop implemented with an MPC.

Applying (7) and (8) into (13), the cost function can be expressed as a function of $v_{x u}(k)$ and $v_{x l}(k)$ as

$$
\begin{aligned}
J_{i}\left(v_{x u}(k), v_{x l}(k)\right) & =p_{1} g_{1}^{2}\left[v_{x u}(k)-v_{x l}(k)+f_{1}(k)\right]^{2} \\
& +p_{2} g_{2}^{2}\left[v_{x u}(k)+v_{x l}(k)+f_{2}(k)\right]^{2}
\end{aligned}
$$

where

$$
g_{1}=\frac{T_{s}}{L_{a r m}+2 L}, g_{2}=\frac{T_{s}}{2 L_{a r m}}
$$

and $f_{1,2}(k)$ are defined as

$$
\begin{aligned}
& f_{1}(k)=2 v_{S x}(k)+\left(R_{\text {arm }}+2 R\right) i_{x}(k)+\frac{i_{x}^{*}(k+1)-i_{x}(k)}{g_{1}} \\
& f_{2}(k)=2 R_{\text {arm }} i_{\text {circ }, x}(k)-V_{d c}+\frac{i_{\text {circ }, x}^{*}(k+1)-i_{\text {circ }, x}(k)}{g_{2}}
\end{aligned}
$$

For the sake of simplicity, setting

$$
\lambda_{1}=p_{1} g_{1}^{2}, \lambda_{2}=p_{2} g_{2}^{2}
$$

then (14) can be reformed as

$$
\begin{array}{r}
J_{i}\left(v_{x u}(k), v_{x l}(k)\right)=\lambda_{1}\left[v_{x u}(k)-v_{x l}(k)+f_{1}(k)\right]^{2}+ \\
\lambda_{2}\left[v_{x u}(k)+v_{x l}(k)+f_{2}(k)\right]^{2} .
\end{array}
$$

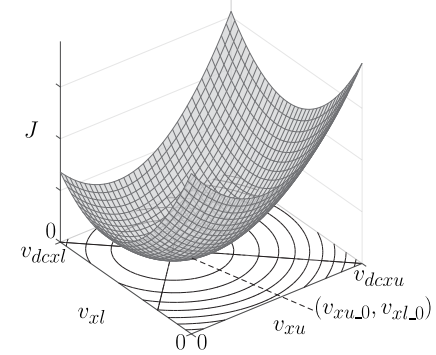

(a)

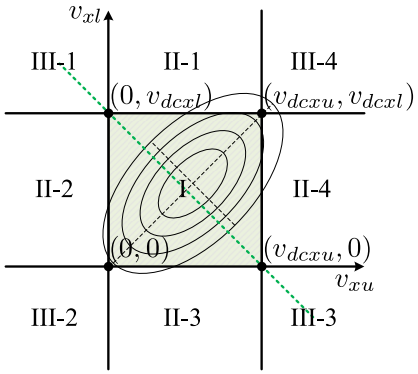

(b)
Fig. 3. Geometrical interpretation. (a) The elliptic paraboloid in orthogonal coordinates. (b) 9 regions where the unconstrained solutions fall.

2) Considering arm energy regulation: To regulate the energy stored in the SMs, the cost function can be defined as follows

$$
J_{w}=p_{3}\left(W_{x \Delta}^{*}-\hat{W}_{x \Delta}\right)^{2}+p_{4}\left(W_{x \Sigma}^{*}-\hat{W}_{x \Sigma}\right)^{2} .
$$

Similar to $J_{i}, J_{w}$ can be arranged into the following form

$$
\begin{aligned}
& J_{w}\left(v_{x u}(k), v_{x l}(k)\right)=\lambda_{3}\left[i_{x u}(k) v_{x u}(k)-i_{x l}(k) v_{x l}(k)\right. \\
& \left.+f_{3}(k)\right]^{2}+\lambda_{4}\left[i_{x u}(k) v_{x u}(k)+i_{x l}(k) v_{x l}(k)+f_{4}(k)\right]^{2}
\end{aligned}
$$

where

$$
\begin{gathered}
\lambda_{3}=p_{3} T_{s}^{2}, \lambda_{4}=p_{4} T_{s}^{2} \\
f_{3}(k)=\frac{W_{x \Delta}(k)-W_{x \Delta}^{*}(k+1)}{T_{s}} \\
f_{4}(k)=\frac{W_{x \Sigma}(k)-W_{x \Sigma}^{*}(k+1)}{T_{s}} .
\end{gathered}
$$

Normally, $J_{w}$ is not applied alone, but together with $J_{i}$ as

$$
J_{i w}=J_{i}+J_{w} .
$$

\section{Optimization Problem}

Within the frame of the MPC, the cost function (either $J_{i}$ or $J_{i w}$ ) has to be minimized to achieve optimal performance. In the previously proposed MPC schemes, the minimization of the cost function is realized by evaluating different voltage levels of $v_{x y}(k)$ in an exhaustive manner, leading to a considerable computational cost. As an alternative proposed in this paper, the cost function can be minimized employing numerical optimization techniques. Thus, optimization problems need to be formulated.

1) Case of $J_{i}$ : Considering optimizing $J_{i}$, it can be inferred from (19) that the cost function reaches the minimum (zero) when and only when the following equations are satisfied

$$
\begin{aligned}
& v_{x u}(k)-v_{x l}(k)+f_{1}(k)=0 \\
& v_{x u}(k)+v_{x l}(k)+f_{2}(k)=0
\end{aligned}
$$

leading to the global solution as

$$
v_{x u \_0}(k)=-\frac{f_{1}(k)+f_{2}(k)}{2}, v_{x l_{-} 0}(k)=\frac{f_{1}(k)-f_{2}(k)}{2} .
$$

To gain a further insight into the cost function, (19) is altered into the following matrix form as follows. For simplicity, the 
time instant $k$ is not explicitly indicated. Throughout the paper, all variables denote those of time instant $k$ unless otherwise stated.

$$
J_{i}\left(\boldsymbol{v}_{\boldsymbol{x} \boldsymbol{y}}\right)=\boldsymbol{v}_{\boldsymbol{x} \boldsymbol{y}}^{T} \boldsymbol{Q}_{\boldsymbol{i}} \boldsymbol{v}_{\boldsymbol{x} \boldsymbol{y}}+2 \boldsymbol{c}_{\boldsymbol{i}}^{T} \boldsymbol{v}_{\boldsymbol{x} \boldsymbol{y}}+d_{i}
$$

where the related variables are defined as follows

$$
\begin{gathered}
\boldsymbol{Q}_{\boldsymbol{i}}=\left[\begin{array}{ll}
q_{i 1} & q_{i 2} \\
q_{i 2} & q_{i 3}
\end{array}\right]=\left[\begin{array}{cc}
\lambda_{1}+\lambda_{2} & -\lambda_{1}+\lambda_{2} \\
-\lambda_{1}+\lambda_{2} & \lambda_{1}+\lambda_{2}
\end{array}\right] \\
\boldsymbol{c}_{\boldsymbol{i}}=\left[\begin{array}{l}
c_{i 1} \\
c_{i 2}
\end{array}\right]=\left[\begin{array}{c}
\lambda_{1} f_{1}+\lambda_{2} f_{2} \\
-\lambda_{1} f_{1}+\lambda_{2} f_{2}
\end{array}\right], \boldsymbol{v}_{\boldsymbol{x y}}=\left[\begin{array}{c}
v_{x u} \\
v_{x l}
\end{array}\right] .
\end{gathered}
$$

Since

$$
\operatorname{det}\left(\boldsymbol{Q}_{\boldsymbol{i}}\right)=4 \lambda_{1} \lambda_{2}>0,
$$

$Q_{i}$ is positive definite. Therefore, (29) is strictly convex with at most one minimum point [27], and (29) represents an elliptic paraboloid in orthogonal coordinates $\left(v_{x u}, v_{x l}, J_{i}\left(v_{x u}, v_{x l}\right)\right)$ as shown in Fig. 3(a). The contour lines of $J_{i}$ are a series of ellipses with the same center point $\left(v_{x u_{-} 0}, v_{x l_{1} 0}\right)$, the same eccentricity, and the same major/minor axis with equation of (26)/(27). As the weighting factor $\lambda_{2}$ increases relative to $\lambda_{1}$, the elliptic paraboloid is stretched along the axis of (27).

However, the arm voltage is bounded as (12). Thus, the unconstrained solution (28) cannot be adopted directly. Since the constrains are linear, the optimization problem to be solved is a typical quadratic programming problem, which can be formulated as

$$
\min _{\boldsymbol{v}_{\boldsymbol{x} \boldsymbol{y}}} J_{i}\left(\boldsymbol{v}_{\boldsymbol{x} \boldsymbol{y}}\right), \quad \text { subject to } \boldsymbol{A} \boldsymbol{v}_{\boldsymbol{x} \boldsymbol{y}} \preceq \boldsymbol{b}
$$

where

$$
\begin{gathered}
\boldsymbol{A}=\left[\begin{array}{cccc}
1 & 0 & -1 & 0 \\
0 & 1 & 0 & -1
\end{array}\right]^{T} \\
\boldsymbol{b}=\left[\begin{array}{llll}
v_{d c x u} & v_{d c x l} & 0 & 0
\end{array}\right]^{T} .
\end{gathered}
$$

2) Case of $J_{i w}$ : Similar to (29) and (30), $J_{w}$ can be rearranged into the matrix form as

$$
J_{w}\left(\boldsymbol{v}_{\boldsymbol{x} \boldsymbol{y}}\right)=\boldsymbol{v}_{\boldsymbol{x} \boldsymbol{y}}^{T} \boldsymbol{Q}_{\boldsymbol{w}} \boldsymbol{v}_{\boldsymbol{x} \boldsymbol{y}}+2 \boldsymbol{c}_{\boldsymbol{w}}^{T} \boldsymbol{v}_{\boldsymbol{x} \boldsymbol{y}}+d_{w}
$$

where

$$
\begin{aligned}
\boldsymbol{Q}_{\boldsymbol{w}}= & {\left[\begin{array}{ll}
q_{w 1} & q_{w 2} \\
q_{w 2} & q_{w 3}
\end{array}\right] } \\
= & {\left[\begin{array}{cc}
i_{x u}^{2}\left(\lambda_{3}+\lambda_{4}\right) & i_{x u} i_{x l}\left(-\lambda_{3}+\lambda_{4}\right) \\
i_{x u} i_{x l}\left(-\lambda_{3}+\lambda_{4}\right) & i_{x l}^{2}\left(\lambda_{3}+\lambda_{4}\right)
\end{array}\right] } \\
& \boldsymbol{c}_{\boldsymbol{w}}=\left[\begin{array}{l}
c_{w 1} \\
c_{w 2}
\end{array}\right]=\left[\begin{array}{c}
i_{x u}\left(\lambda_{3} f_{3}+\lambda_{4} f_{4}\right) \\
i_{x l}\left(-\lambda_{3} f_{3}+\lambda_{4} f_{4}\right)
\end{array}\right]
\end{aligned}
$$

From (25), (29) and (34), $J_{i w}$ can be derived as

$$
J_{i w}\left(\boldsymbol{v}_{\boldsymbol{x} \boldsymbol{y}}\right)=\boldsymbol{v}_{\boldsymbol{x} \boldsymbol{y}}^{T} \boldsymbol{Q}_{\boldsymbol{i w}} \boldsymbol{v}_{\boldsymbol{x} \boldsymbol{y}}+2 \boldsymbol{c}_{\boldsymbol{i w}}^{T} \boldsymbol{v}_{\boldsymbol{x} \boldsymbol{y}}+d_{i w}
$$

where

$$
\begin{gathered}
\boldsymbol{Q}_{\boldsymbol{i w}}=\left[\begin{array}{ll}
q_{i w 1} & q_{i w 2} \\
q_{i w 2} & q_{i w 3}
\end{array}\right]=\boldsymbol{Q}_{\boldsymbol{i}}+\boldsymbol{Q}_{\boldsymbol{w}} \\
\boldsymbol{c}_{\boldsymbol{i w}}=\left[\begin{array}{l}
c_{i w 1} \\
c_{i w 2}
\end{array}\right]=\boldsymbol{c}_{\boldsymbol{i}}+\boldsymbol{c}_{\boldsymbol{w}}
\end{gathered}
$$

The problem to be optimized is

$$
\min _{\boldsymbol{v}_{\boldsymbol{x} \boldsymbol{y}}} J_{i w}\left(\boldsymbol{v}_{\boldsymbol{x} \boldsymbol{y}}\right), \quad \text { subject to } \boldsymbol{A} \boldsymbol{v}_{\boldsymbol{x} \boldsymbol{y}} \preceq \boldsymbol{b} .
$$

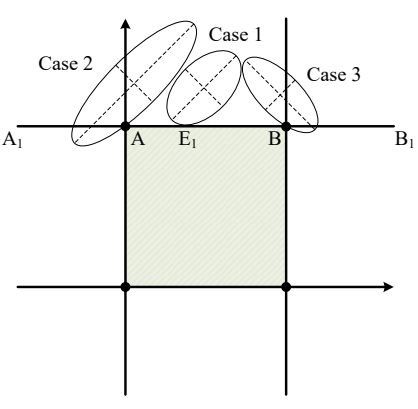

(a)

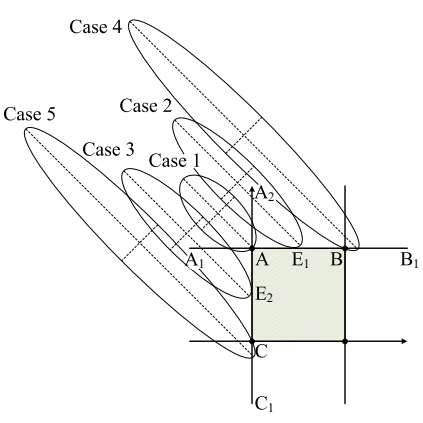

(b)
Fig. 4. Solution for Regions II and III.

Since

$$
\begin{array}{r}
\operatorname{det}\left(\boldsymbol{Q}_{\boldsymbol{i w}}\right)=4 \lambda_{1} \lambda_{2}+4 i_{x u}^{2} i_{x l}^{2} \lambda_{3} \lambda_{4}+i_{x}^{2}\left(\lambda_{1} \lambda_{3}+\lambda_{2} \lambda_{4}\right) \\
+4 i_{\text {circ }, x}^{2}\left(\lambda_{1} \lambda_{4}+\lambda_{2} \lambda_{3}\right)>0
\end{array}
$$

The optimization problem (38) is a positive-definite quadratic programming problem as (32).

\section{SOLVING FOR ARM VOLTAGE REFERENCES USING QUADRATIC PROGRAMMING}

\section{A. Rigorous Solution}

1) Solution of $J_{i}$ : As mentioned above, the global solution of the unconstrained version of (32) can be obtained as $\left(v_{x u_{-} 0}, v_{x l_{-} 0}\right)$ determined from (28). As an equivalent measure, $\left(v_{x u_{-} 0}, v_{x l_{-} 0}\right)$ can be obtained from

$$
\nabla J_{i}\left(\boldsymbol{v}_{\boldsymbol{x} \boldsymbol{y}_{-} \mathbf{0}}\right)=2\left(\boldsymbol{Q}_{\boldsymbol{i}} \boldsymbol{v}_{\boldsymbol{x} \boldsymbol{y}_{-} \mathbf{0}}+\boldsymbol{c}_{\boldsymbol{i}}\right)=\mathbf{0} .
$$

Considering the constraints, several techniques are available such as the active-set, interior-point or first-order methods, which normally require multiple steps of iterations and/or solutions of a linear system of equations [28]. Since the feasible set of (32) is only two-dimensional, the solution can be derived explicitly from geometrical interpretation.

According to the constraints of (32), the Euclidean plane of $\left(v_{x u}, v_{x l}\right)$ can be divided into 9 regions as shown in Fig. 3(b). Apparently $\left(v_{x u_{-} 0}, v_{x l_{0} 0}\right)$ is not the solution of (32) when it falls outside region I (the feasible region). In that case, the optimization problem (32) is equivalent to looking for the first point in region I that touches the elliptical contour lines expanding from the center $\left(v_{x u_{-} 0}, v_{x l_{-} 0}\right)$. To solve (32), the 9 regions shown in Fig. 3(b) can be divided into three types: I, II and III. For each group, the procedures of obtaining the solution are explained as follows:

1) $\left(v_{x u_{-} 0}, v_{x l_{-} 0}\right)$ falls into region $\mathrm{I}$ :

In this case, the solution of (32) is (28), as shown in Fig. 3(b).

2) $\left(v_{x u_{-} 0}, v_{x l_{-} 0}\right)$ falls into region II:

Region II- 1 is studied here as an example because the other three regions (II-2,3,4) are similar. In this situation, the optimum of (32) lies on segment $\mathrm{AB}$ and can be divided into three cases as illustrated in Fig. 4(a). Firstly, the point $E_{1}$ on the segment $A_{1} B_{1}$ that first touches the 
expanding contours (i.e., the tangent point) is obtained by solving

$$
\left.\frac{\partial J_{i}}{\partial v_{x u}}\right|_{v_{x l_{-} E 1}=v_{d c x l}}=2\left(q_{i 1} v_{x u_{-} E 1}+q_{i 2} v_{d c x l}+c_{i 1}\right)=0
$$

leading to

$$
v_{x u_{-} E 1}=-\frac{q_{i 2} V_{d c x l}+c_{i 1}}{q_{i 1}}
$$

which specifies the coordinate of $\mathrm{E}_{1}$ as $\left(v_{x u_{-} E 1}, v_{d c x l}\right)$. Then, the solution of (32) can be achieved as follows:

a) Case 1: if $\mathrm{E}_{1}$ is on $\mathrm{AB}\left(0 \leq v_{x u_{-} E 1} \leq v_{d c x u}\right)$, the solution is $\mathrm{E} 1$.

b) Case 2: if $\mathrm{E}_{1}$ is on $\mathrm{AA}_{1}\left(v_{x u_{-} E 1}<0\right)$, the solution is A.

c) Case 3: if $\mathrm{E}_{1}$ is on $\mathrm{BB}_{1}\left(v_{x u_{-} E 1}>v_{d c x u}\right)$, the solution is $\mathrm{B}$.

3) $\left(v_{x u_{-} 0}, v_{x l_{0} 0}\right)$ falls into region III:

Region III- 1 is studied here as an example because the other three regions (III-2,3,4) are similar. In this situation, the optimum of (32) lies on segment $\mathrm{AB}$ or $\mathrm{AC}$ and can be divided into five cases as illustrated in Fig. 4(b). Firstly, the tangent points on the line $\mathrm{A}_{1} \mathrm{~B}_{1}$ and $\mathrm{A}_{2} \mathrm{C}_{1}$ that first touch the expanding contour lines are obtained as $E_{1}$ and $E_{2}$ in a similar manner with the case of region II. Then, the solution of (32) can be achieved as follows:

a) Case 1: if $E_{1}$ is on $\mathrm{AA}_{1}$ and $\mathrm{E}_{2}$ is on $\mathrm{AA}_{2}$, the solution is $\mathrm{A}$.

b) Case 2: if $E_{1}$ is on $A B$, the solution is $E_{1}$.

c) Case 3: if $E_{2}$ is on $A C$, the solution is $E_{2}$.

d) Case 4: if $E_{1}$ is on $B B_{1}$, the solution is $B$.

e) Case 5: if $\mathrm{E}_{2}$ is on $\mathrm{CC}_{1}$, the solution is $\mathrm{C}$.

The complete solution for all the regions are provided in the appendix.

2) Solution of $J_{i w}$ : The solution of (38) can be obtained in a similar manner since it presents an objective function with the same constraints and structure introduced in (32). Similar to (40), the global optimal solution (without constraints) should satisfy

$$
\nabla J_{i w}\left(\boldsymbol{v}_{\boldsymbol{x} \boldsymbol{y}_{-} \mathbf{0}}\right)=2\left(\boldsymbol{Q}_{\boldsymbol{i w}} \boldsymbol{v}_{\boldsymbol{x} \boldsymbol{y}_{-} \mathbf{0}}+\boldsymbol{c}_{\boldsymbol{i w}}\right)=\mathbf{0}
$$

leading to

$$
\boldsymbol{v}_{\boldsymbol{x} \boldsymbol{y}_{\_} \mathbf{0}}=\left[\begin{array}{ll}
v_{x u_{-} 0} & v_{x l_{-} 0}
\end{array}\right]^{T}=-\boldsymbol{Q}_{\boldsymbol{i w} \boldsymbol{w}}^{-1} \boldsymbol{c}_{\boldsymbol{i w}} .
$$

Then, the procedures described above for solving (32) can be repeated for (38).

In terms of implementation, the above-described scheme of region assignation and solution determination can be well designed to minimize the involved calculation cost. The corresponding flowchart with the least possible calculations is outlined in Fig. 5.

\section{B. Simplified Solution}

Although the rigorous procedures do not involve complex calculations, (32) and (38) can be solved in a simpler manner to accelerate the implementation. During normal operation of

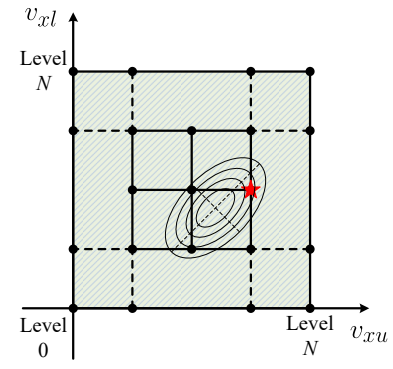

(a)

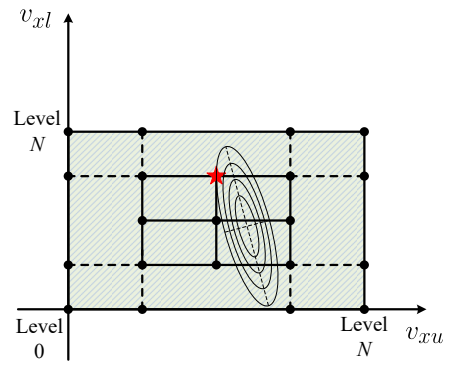

(b)
Fig. 6. Determination of optimal arm voltage levels of (a) a normal case. (b) an abnormal case.

the MMC, the sum of arm voltages of each phase should always be kept close to the DC-voltage as

$$
v_{x u}+v_{x l} \approx V_{d c}
$$

in order to avoid generating high circulating current. Thus, all $\left(v_{x u_{0} 0}, v_{x l_{-} 0}\right)$ should fall close to the diagonal line shown in Fig. 3(b) (the green dotted line) even if they are outside region I. In addition, the contour lines of the cost function are normally not extremely long-and-narrow. Considering the above factors, a simplified solution of (32) and (38) can be obtained by simply passing the unconstrained solution through a saturation block as

$$
v_{x y}^{*}= \begin{cases}0 & v_{x y_{\_} 0}<0 \\ v_{x y_{-} 0} & 0 \leq v_{x y_{\_} 0} \leq v_{d c x y} \\ v_{d c x y} & v_{x y_{\_} 0}>v_{d c x y}\end{cases}
$$

which is equivalent to the rigorous solution with $\lambda_{1}=\lambda_{2}$ and $\lambda_{3}=\lambda_{4}=0$, forcing the elliptic contour lines mentioned above to evolve into circular contour lines.

\section{Determination of ARM VOlTAGE LEVEL AND GATE SIGNALS}

From the references of arm voltages (denoted as $v_{x y}^{*}$ ) obtained from the proposed method, the SM gate signals are determined applying a specific modulation method, such as the phase-shift/level-shift PWM or the NLC. Since the objective of this paper is to determine the optimal arm voltage level of the MMC, the variable rounding level control (VRLC) pseudomodulation scheme proposed in [26] is adopted. The basic principle of VRLC is to select ceiling() or floor() rounding function after normalizing the arm voltage reference as

$$
N_{x y}^{*}=\text { ceiling/floor }\left(\frac{v_{x y}^{*}}{v_{d c x y} / N}\right)
$$

and the decision is made by the FCS-MPC technique. Further details of the VRLC method can be found in [26]. It is worth noting that if $J_{i}$ is employed without arm-energy regulating terms, $v_{d c x y}=V_{d c}$ has to be adopted as indirect capacitorvoltage regulation for stability [16].

To facilitate the understanding, the above concept is illustrated in Fig. 6. The whole feasible region is divided by $(N+1)^{2}$ candidates into $N^{2}$ units. In the case of Fig. 6(a), (47) adopts $v_{d c x y}=V_{d c}$ for normalization and thus each unit 


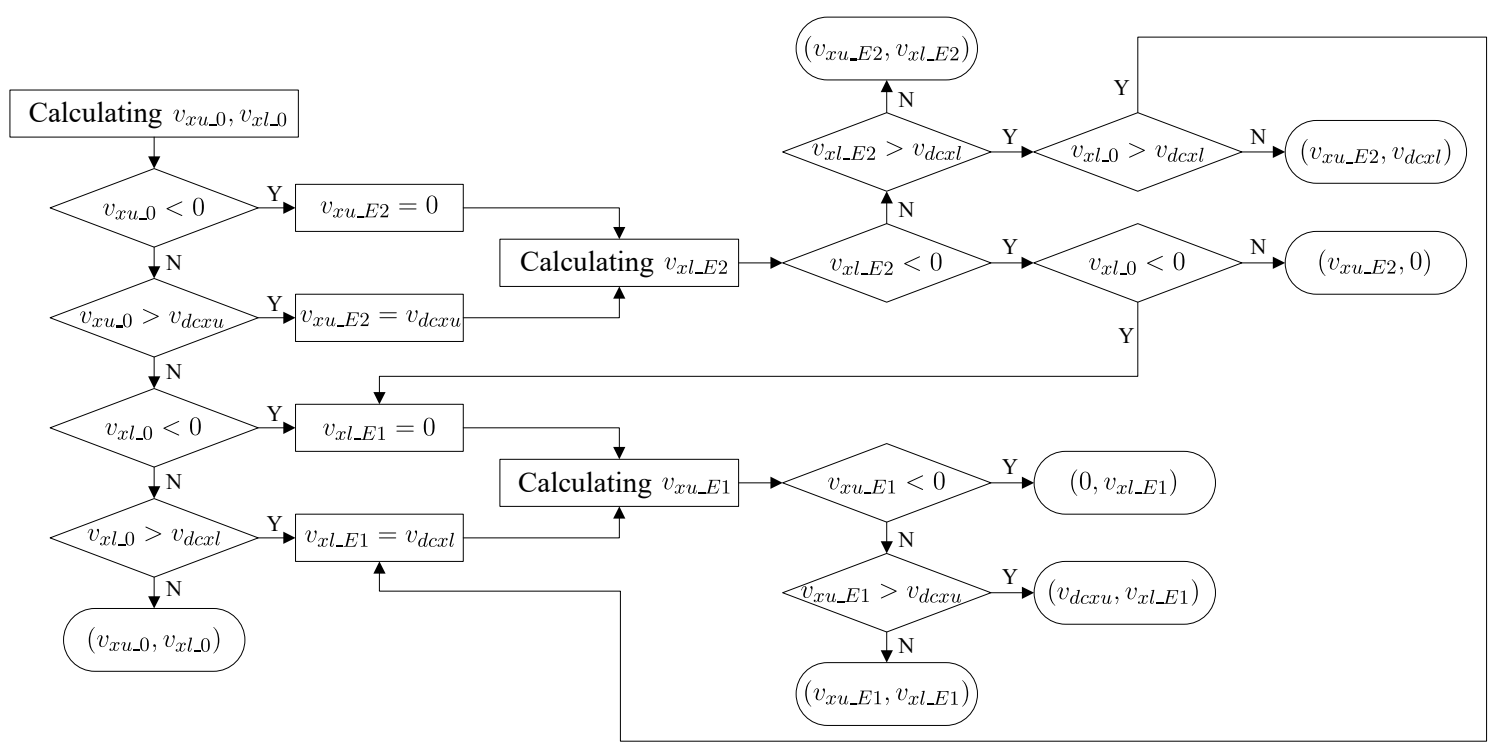

Fig. 5. Flowchart of region-search and solution-determination scheme.

is a square. In addition, $J_{i}$ is adopted as the cost function leading to an angle of $\pi / 4$ between the major/minor axis of the contour lines and the $v_{x y}$ axis as inferred from (26) and (27). Therefore, it can be deduced that in this case, the final solution of $N_{x y}^{*}$ can be obtained by just evaluating the four vertices of the unit where the unconstrained solution (the center of the elliptical contour lines) falls. The optimal candidate is marked by the red-star icon. It is important to mention that the optimal candidate obtained by the proposed technique is identical to that determined by the conventional MPC scheme [15], which however has to evaluate all the $(N+1)^{2}$ possible candidates.

In the case of Fig. 6(b), the SM capacitor voltages are used for (47), and the $N^{2}$ units are thus rectangular but not necessarily square. In addition, $J_{i w}$ is adopted as the cost function (with $\lambda_{3,4} \neq 0$ ), making the major/minor axis of the contour lines form an angle different from $\pi / 4$ with the $v_{x y}$ axis. In this case, the reduced four-candidate-evaluation scheme adopted by the VRLC technique may not be applicable, and the solution may be outside the unit as shown in Fig. 6(b).

In a real MMC implementation, however, $v_{d c x u} \approx v_{d c x l} \approx$ $V_{d c}$ since the capacitor voltage ripples should be much lower than $V_{d c}$. Additionally, the incorporation of $J_{w}$ for arm energy regulation is auxiliary and should not change the slope of the major/axis (of the contour lines) to a large extent. Therefore, the assumption of Fig. 6(a) can be made and the VRLC technique can be applied without sacrificing performance compared with the original method evaluating all $(N+1)^{2}$ candidates [15].

The last step is to select the individual switched-on SMs within each arm. The conventional sorting scheme [1] is employed to undertake the SM-capacitor-voltage-balancer (SMCVB) task as follows

1) if the corresponding arm current is positive, the SMs with the lowest capacitor voltages will be switched on.

2) if the corresponding arm current is negative, the SMs with the highest capacitor voltages will be switched on.

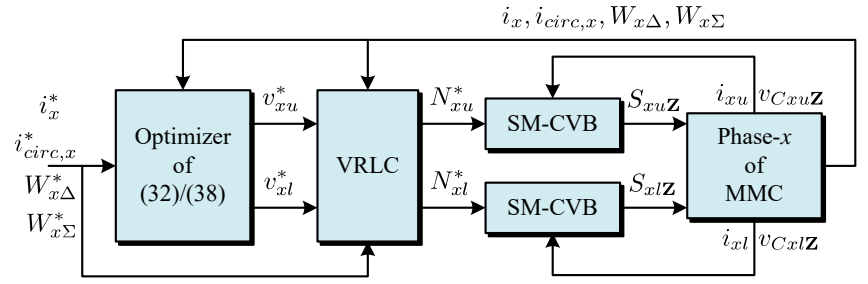

Fig. 7. Block diagram of the entire proposed method $(\mathbf{Z}=\{1,2, \ldots, N\})$.

The entire control scheme of phase- $x$ is outlined in Fig. 7, which starts from a set of given references since their determination is not the focus of this paper. For a specified application, the proposed method can be easily integrated by simply introducing the corresponding outer-loop controllers that lead to the phase-current references $i_{x}^{*}$, such as the speed/torque and flux controllers in motor drive applications, active- and reactive-power controllers in grid-connected applications, etc. A detailed review of outer-loop-controller design can be found in [20]. In terms of the setting of circulating-current reference $i_{c i r c, x}^{*}, i_{d c} / 3$ or its DC component (determined based on the power balance of the MMC between its $\mathrm{AC}$ side and DC side) is commonly adopted [15], [18]. In addition, some harmonics can be included for various purposes [29]. For further enhancing the regulation of SM-capacitor voltages, $i_{c i r c, x}^{*}$ can be given by extra arm-voltage controllers [25].

The proposed technique can also be incorporated into abnormal operations. As an example, in case of unbalanced grid conditions, negative-sequence component needs to be included in $i_{x}^{*}$ to maintain a constant active- and reactive-power transfer and $i_{c i r c, x}^{*}$ is recommended to be set as a pure DC value (instead of $i_{d c} / 3$ which contains ripples) for the regulation of zero-sequence and unbalanced circulating current [14], [23]. In addition, another issue in this scenario is that the commonmode voltage of the AC-side neutral point has to be considered in the phase-current model (1). 


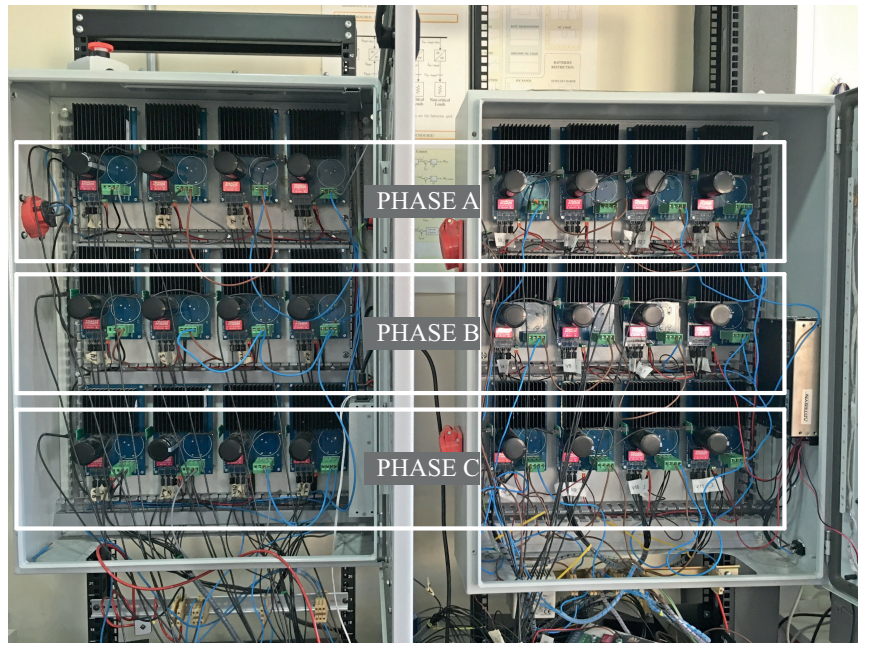

Fig. 8. The experimental setup (left/right cabinet: upper/lower arms).

TABLE I

PARAMETERS OF THE MMC-BASED SYSTEMS UNDER STUDY.

\begin{tabular}{c|c|c}
\hline \hline \multirow{2}{*}{ Parameter } & \multicolumn{2}{|c}{ Value in } \\
\cline { 2 - 3 } & Experiment & Simulation \\
\hline \hline Number of SMs per arm, $N$ & 4 & 200 \\
SM capacitance, $C$ & $1 \mathrm{mF}$ & $20 \mathrm{mF}$ \\
Arm inductance, $L_{\text {arm }}$ & $15 \mathrm{mH}$ & $40 \mathrm{mH}$ \\
Arm resistance, $R_{\text {arm }}$ & $0.5 \Omega$ & $0.1 \Omega$ \\
DC-link voltage, $V_{d c}$ & $180 \mathrm{~V}$ & $128 \mathrm{kV}$ \\
Grid voltage, peak value of $v_{S x}$ & 0 & $57 \mathrm{kV}$ \\
Load/grid inductance, $L$ & $15 \mathrm{mH}$ & $5 \mathrm{mH}$ \\
Load/grid resistance, $R$ & $20.5 \Omega$ & $0.1 \Omega$ \\
AC frequency, $f$ & $50 \mathrm{~Hz}$ & $50 \mathrm{~Hz}$ \\
Sampling/control time, $T_{s}$ & $0.2 \mathrm{~ms}$ & $0.2 \mathrm{~ms}$ \\
\hline \hline
\end{tabular}

Remark: Since only four candidates are evaluated per phase per sampling period (regardless of $N$ ), the proposed method presents a significant advantage in terms of computational burden compared with the conventional FCS-MPC schemes which evaluate a great number of (at least proportional to $N$ ) candidates [14]-[18]. Taking into account the calculation of $v_{x y_{-} 0}$, the worst-case implementation of Fig. 5 (both $v_{x u_{-} E 1}$ and $v_{x l_{-} E 2}$ are calculated) encompasses operations of 5 additions/subtractions, 8 multiplications, 4 divisions and 7 value comparisons. Additionally, every single evaluation of the cost function encompasses operations of 5 additions/subtractions, 8 multiplications and 1 value comparison. Thus, the proposed approach achieves much lower computational cost compared with the exhaustive MPC method. This advantage is achieved even if the MMC has a reduced number of SMs and becomes a critical issue when the number of SMs is large.

\section{EXPERIMENTAL ANALYSIS}

To evaluate the performance of the proposed method, a three-phase MMC setup with $N=4$ SMs per arm (shown in Fig. 8) is employed to conduct the experimental tests. Important system parameters are summarized in Table I. The proposed control scheme is implemented on the dSPACE 1007 platform. The one-sampling-period delay is compensated with the technique presented in [30], which applies an extra step of prediction before employing the proposed method in the second-step prediction.

\section{A. Steady-State Performance}

The steady-state tests are evaluated first. $i_{x}^{*}$ is assigned a balanced three-phase sinusoidal waveform with peak value of $3.7 \mathrm{~A}$. $i_{c i r c, x}^{*}$ is set as a constant value of $0.86 \mathrm{~A}$, which is determined manually (ideal DC reference [14] plus a slight adjustment accounting for the converter losses) instead of using a total-arm-energy controller [7] in order to observe the current tracking performance. $W_{x \Delta}^{*}$ is set as zero and $W_{x \Sigma}^{*}$ is set as $C N\left(V_{d c} / N-0.8\right)^{2}$ considering the influence of IGBT voltage drop as $0.8 \mathrm{~V}$.

The performance of the proposed techniques including both the rigorous and simplified solutions is shown in Fig. 9 and Fig. 10, while the emphasis is given to phase- $a$. For comparative purposes, the conventional FCS-MPC method [15] is also tested as an optimal-performance reference since it evaluates all $(N+1)^{2}$ insertion-index combinations in each phase. All tests in Fig. 9 evaluate the cost function $J_{i}$ with $\lambda_{1}=1$, $\lambda_{2}=0.25$ while adopting $v_{d c x y}=V_{d c}$ for stability (though a perfect regulation of arm energy is not guaranteed as shown in the unbalanced capacitor voltage waveforms), and those in Fig. 10 evaluate the cost function $J_{i w}$ with $\lambda_{1}=1, \lambda_{2}=0.25$, $\lambda_{3}=1 \times 10^{-3}, \lambda_{4}=1 \times 10^{-4}$.

It can be observed that in both groups of tests, the proposed methods (both the rigorous and simplified solutions) exhibit similar performance with the conventional FCS-MPC method in terms of the phase current quality, circulating current ripple, and the waveforms of phase voltage and capacitor voltages. For a quantitative comparison, the THD (\%, up to the 50th harmonic) of the phase current, the RMS value (A) of the circulating current ripple, and the average switching frequency (Hz) of all the SMs of phase- $a$ applying the different methods are summarized in Table II. More tests with different weighting factors are added for a more complete analysis. In all cases, both proposed solutions present almost identical results to the conventional exhaustive FCS-MPC method.

\section{B. Dynamic Response}

The dynamic performance of the above-mentioned methods is summarized in Fig. $11\left(J_{i}\right)$ and Fig. $12\left(J_{i w}\right)$. In the transient, the amplitude of $i_{x}^{*}$ is reduced to half $(1.85 \mathrm{~A})$ and $i_{\text {circ }, x}^{*}$ is reduced to 0.215 A correspondingly. Fast dynamics in terms of phase currents and circulating current (reflected by the DC-link current $i_{d c}$ ) are achieved in all tests. Similar to the steady-state tests, both proposed solutions present similar results with the conventional exhaustive control scheme.

\section{Computational Burden Analysis}

It is important to highlight that in terms of complexity, the proposed solutions involve much less computational burden than the conventional FCS-MPC scheme. The execution time $(\mu \mathrm{s})$ of the determination of insertion indexes (neglecting the 


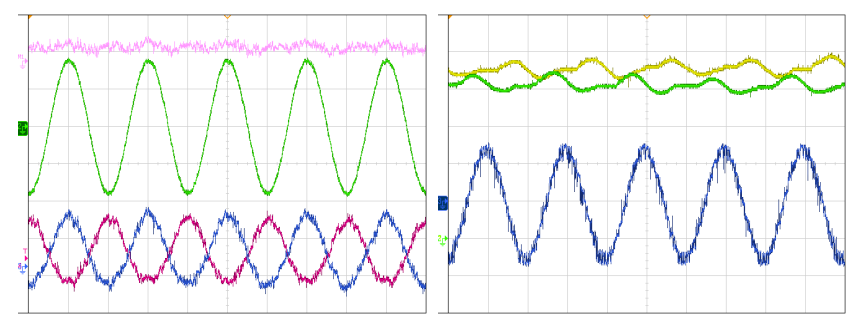

(a) Conventional method

(b) Conventional method

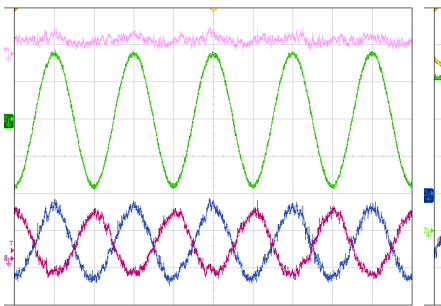

(c) Rigorous solution

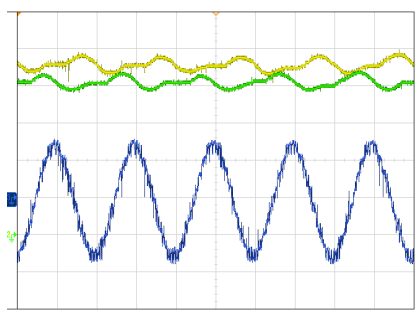

(d) Rigorous solution

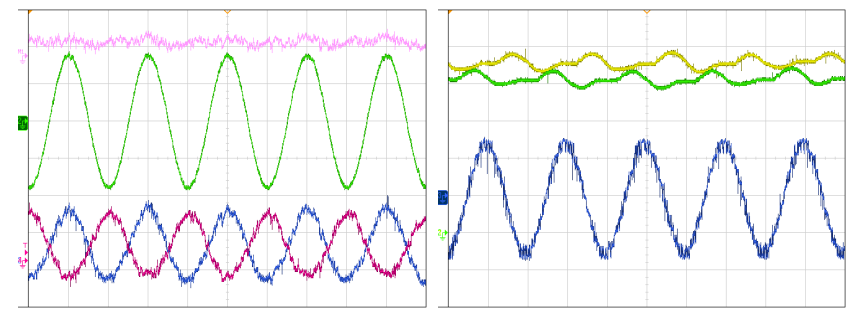

(e) Simplified solution

(f) Simplified solution

Fig. 9. Steady-state performance of different methods using $J_{i}$ (with $\left.v_{d c x y}=V_{d c}\right):(\mathrm{a})(\mathrm{c})(\mathrm{e})$ show $i_{a}($ green, $2.00 \mathrm{~A} /), i_{c i r c, a}($ pink, $2.00 \mathrm{~A} /)$, $i_{a u}$ (blue, $2.00 \mathrm{~A} /$ ), $i_{a l}$ (red, $2.00 \mathrm{~A} /$ ); (b)(d)(f) show $v_{C a u 2}$ (yellow, 10.0 $\mathrm{V} /), v_{\text {Cal2 }}($ green, $10.0 \mathrm{~V} /), v_{a}$ (blue, $\left.50.0 \mathrm{~V} /\right)$; time $(10.00 \mathrm{~ms} /)$.

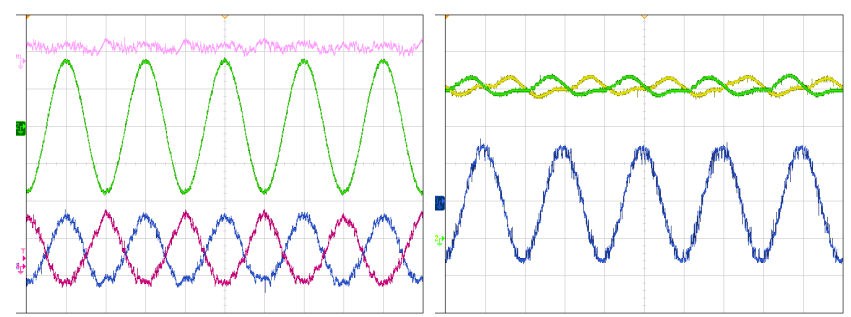

(a) Conventional method

(b) Conventional method

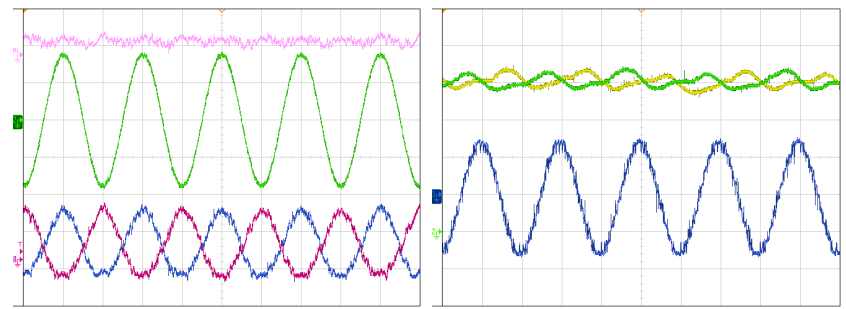

(c) Rigorous solution

(d) Rigorous solution

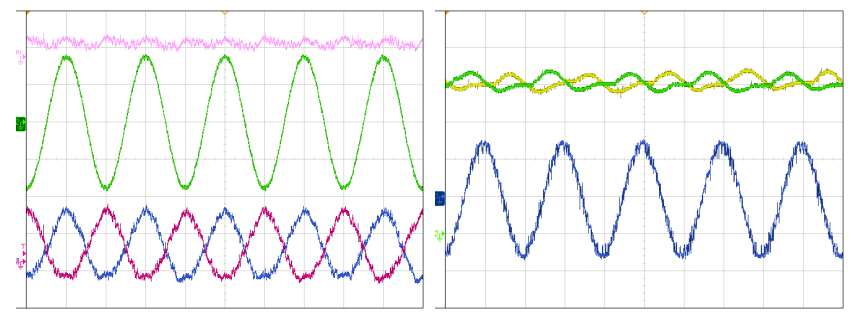

(e) Simplified solution

(f) Simplified solution

Fig. 10. Steady-state performance of different methods using $J_{i w}$ : (a)(c)(e) show $i_{a}$ (green, $2.00 \mathrm{~A} /$ ), $i_{c i r c, a}$ (pink, $2.00 \mathrm{~A} /$ ), $i_{a u}$ (blue, $2.00 \mathrm{~A} /$ ), $i_{a l}$ (red, $2.00 \mathrm{~A} /$ ); (b)(d)(f) show $v_{\text {Cau } 2}$ (yellow, $10.0 \mathrm{~V} /$ ), $v_{\text {Cal } 2}$ (green, 10.0 $\mathrm{V} /), v_{a}$ (blue, $\left.50.0 \mathrm{~V} /\right)$; time $(10.00 \mathrm{~ms} /)$.

TABLE II

SUMMARY OF STEADY-STATE PERFORMANCE OF DIFFERENT METHODS IN EXPERIMENTAL TESTS.

\begin{tabular}{|c|c|c|c|c|c|c|c|c|c|c|c|c|c|c|}
\hline \multirow{3}{*}{$\begin{array}{c}\text { Cost function } \\
J_{i} \\
\end{array}$} & \multirow{2}{*}{\multicolumn{2}{|c|}{ Weighting factors }} & \multicolumn{4}{|c|}{ Conventional method } & \multicolumn{4}{|c|}{ Rigorous solution } & \multicolumn{4}{|c|}{ Simplified solution } \\
\hline & & & \multirow{2}{*}{$\begin{array}{l}\text { THD } \\
1.81 \\
\end{array}$} & \multirow{2}{*}{$\begin{array}{l}\text { RMS } \\
0.190 \\
\end{array}$} & \multirow{2}{*}{$\begin{array}{c}f_{s w} \\
1196 \\
\end{array}$} & \multirow{2}{*}{$\begin{array}{l}t_{e x} \\
\mathbf{4 . 3 2} \\
\end{array}$} & \multirow{2}{*}{$\begin{array}{l}\text { THD } \\
1.81 \\
\end{array}$} & \multirow{2}{*}{$\begin{array}{l}\text { RMS } \\
0.187 \\
\end{array}$} & \multirow{2}{*}{$\begin{array}{c}f_{s w} \\
1197 \\
\end{array}$} & \multirow{2}{*}{$\begin{array}{l}t_{e x} \\
1.49 \\
\end{array}$} & \multirow{2}{*}{$\begin{array}{l}\text { THD } \\
1.80 \\
\end{array}$} & \multirow{2}{*}{$\begin{array}{l}\text { RMS } \\
0.192 \\
\end{array}$} & \multirow{2}{*}{$\begin{array}{l}f_{s w} \\
1199 \\
\end{array}$} & \multirow{2}{*}{$\begin{array}{l}t_{e x} \\
1.45 \\
\end{array}$} \\
\hline & $\lambda_{1}=1$ & $\lambda_{2}=0.25$ & & & & & & & & & & & & \\
\hline \multirow{3}{*}{$J_{i w}$} & \multirow{3}{*}{$\begin{array}{c}\lambda_{1}=1 \\
\lambda_{3}=1 \times 10^{-3} \\
\lambda_{4}=1 \times 10^{-4}\end{array}$} & $\lambda_{2}=0.25$ & 1.57 & 0.177 & 1070 & 4.34 & 1.55 & 0.175 & 1069 & 1.50 & 1.53 & 0.173 & 1069 & 1.44 \\
\hline & & $\lambda_{2}=1$ & 2.02 & 0.131 & 1081 & 4.35 & 2.02 & 0.130 & 1082 & 1.49 & 2.06 & 0.126 & 1080 & 1.45 \\
\hline & & $\lambda_{2}=4$ & 2.87 & 0.126 & 1179 & 4.36 & 2.92 & 0.123 & 1180 & 1.49 & 2.88 & 0.126 & 1178 & 1.45 \\
\hline
\end{tabular}

common part of the three methods such as calculating the matrix of cost function, delay compensation, etc.) of different tests are summarized in Table II. Both proposed solutions present much lower computational cost than the conventional exhaustive technique, which coincides with the analysis of calculation time in section IV. In addition, it is important to notice that the proposed rigorous solution presents only slightly higher calculation than the simplified solution. In any case, the potential reduction of the computational burden of both proposed methods becomes significant considering the real MMC applications with much higher number of SMs. This would permit to increase the sampling frequency of the MMC leading to improved output waveform quality. Also, it would permit to define more complex cost functions including extra control terms to improve the MMC performance.

\section{Advantage of Rigorous Solution}

In all the tests above, both the rigorous and simplified solutions show a similar behavior. However, in certain conditions where the transient of circulating current is dramatic, the advantages of the rigorous solution can be observed. An example of harmonic injection of circulating current is investigated. Fig. 13 shows the performances of different methods with $J_{i w}$ $\left(\lambda_{1}=1, \lambda_{2}=4, \lambda_{3}=5 \times 10^{-3}, \lambda_{4}=1 \times 10^{-3}\right)$. A negativesequence second harmonic is injected in the circulating current, which is eliminated from $i_{c i r c, x}^{*}$ during the transient. In steady state, all the three methods exhibit similar quality of circulating current injection. In the transient, all cases show fast dynamic, while a slightly higher distortion of $i_{a}$ can be observed in Fig. 13(a) and Fig. 13(b). To further investigate the 


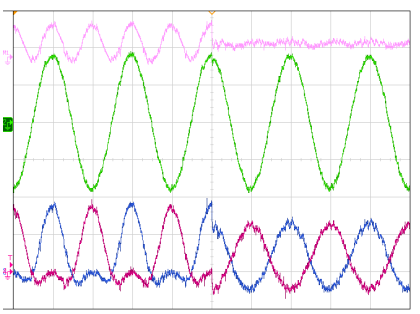

(a) Conventional method

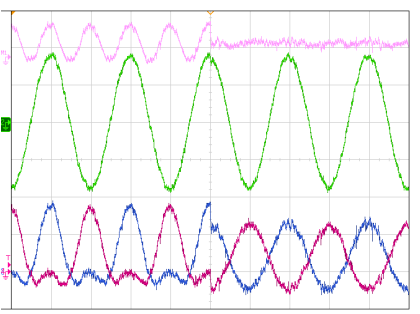

(b) Rigorous solution

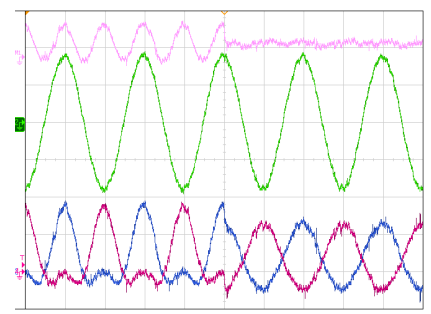

(c) Simplified solution

Fig. 13. Performance evaluation of different methods (using $J_{i w}$ ) in the circulating-current-injection tests: $i_{c i r c, a}$ (pink, $2.00 \mathrm{~A} /$ ), $i_{a}$ (green, $\left.2.00 \mathrm{~A} /\right), i_{a u}$ (blue, $2.00 \mathrm{~A} /), i_{a l}(\mathrm{red}, 2.00 \mathrm{~A} /)$; time $(10.00 \mathrm{~ms} /)$.

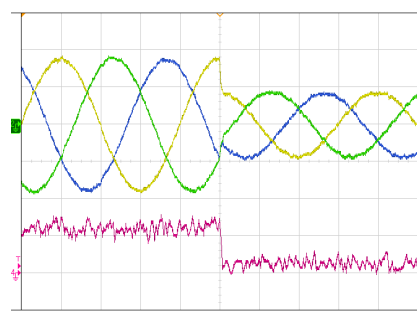

(a) Conventional method

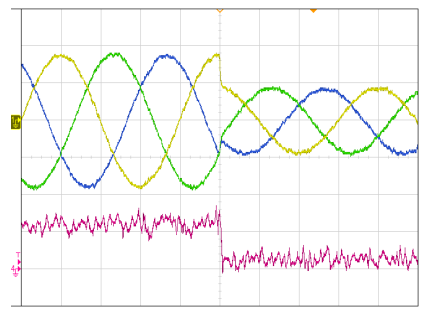

(b) Rigorous solution

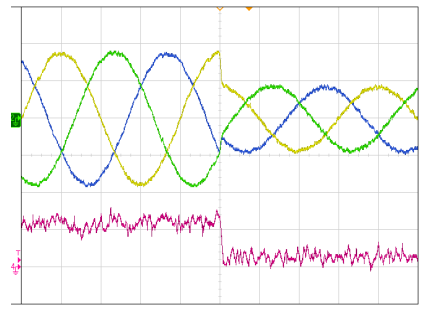

(c) Simplified solution

Fig. 11. Dynamic performance of different methods using $J_{i}$ (with $\left.v_{d c x y}=V_{d c}\right): i_{a}$ (yellow, $2.00 \mathrm{~A} /$ ), $i_{b}$ (green, $2.00 \mathrm{~A} /$ ), $i_{c}$ (blue, $2.00 \mathrm{~A} /$ ), $i_{d c}(\mathrm{red}, 2.00 \mathrm{~A} /)$; time $(5.000 \mathrm{~ms} /)$.

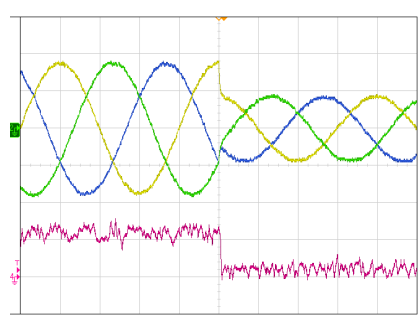

(a) Conventional method

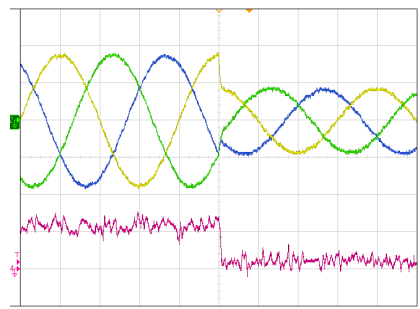

(b) Rigorous solution

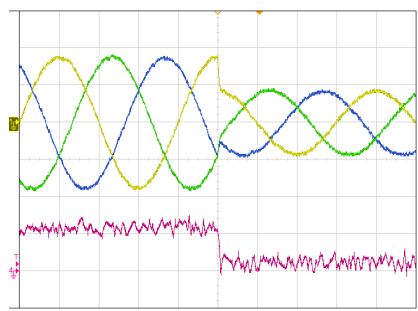

(c) Simplified solution

Fig. 12. Dynamic performance of different methods using $J_{i w}: i_{a}$ (yellow, $2.00 \mathrm{~A} /$ ), $i_{b}$ (green, $2.00 \mathrm{~A} /$ ), $i_{c}$ (blue, $2.00 \mathrm{~A} /), i_{d c}($ red, $2.00 \mathrm{~A} /$ ); time $(5.000 \mathrm{~ms} /)$.

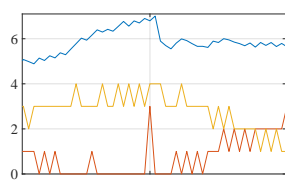

(a) Conventional method

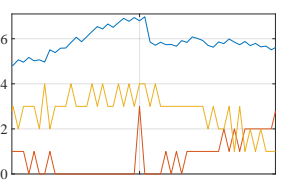

(b) Rigorous solution

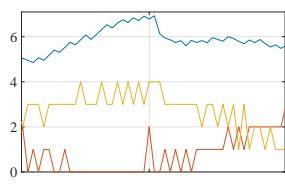

(c) Simplified solution

Fig. 14. Zoom-in (0.01s) of the transient of Fig. 13 using the data from ControlDesk: $i_{c i r c, a}$ (blue), $N_{x u}^{*}$ (red), $N_{x l}^{*}$ (yellow).

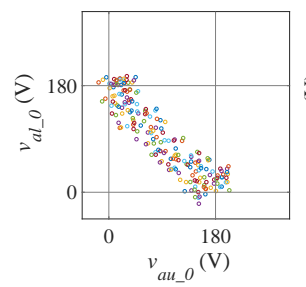

(a) Test of Fig. 10(c)

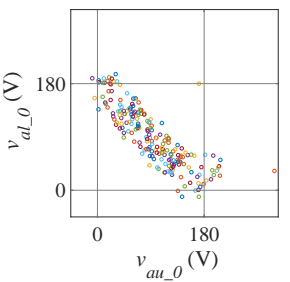

(b) Test of Fig. 12(b)

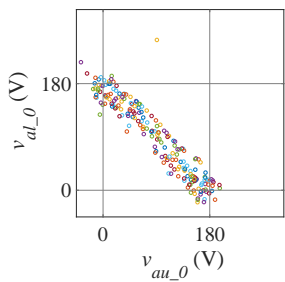

(c) Test of Fig. 13(b)
Fig. 15. Distribution of $v_{a u \_0}, v_{a l_{\_} \_}$during $0.04 \mathrm{~s}$ of different tests.

transient, experimental data from dSPACE (ControlDesk) are employed and the insertion indexes along with the circulating current in reaction to the transient are illustrated in Fig. 14. From the zoom-in figures, it can be clearly observed that the rigorous solution and the conventional exhaustive FCS-MPC show faster dynamic than the proposed simplified solution. In Fig. 14(a) and Fig. 14(b) $N_{a u}^{*}$ increases one extra level compared with the result in Fig. 14(c) to adapt quickly to the transient. Thus, the proposed rigorous solution exhibits better performance than the simplified solution since the circulating current tracking is put in the first place.

\section{E. Visualization of Unconstrained Solutions}

For visualization, the distribution of all $\left(v_{a u_{-} 0}, v_{a l_{-} 0}\right)$ of the proposed rigorous solution during different tests are illustrated in Fig. 15. It can be observed in Fig. 15(a) that all solutions fall close to the diagonal line in the steady state, including those outside region I. Thus, the weighting factors have little impact on the solution of $v_{x y}^{*}$. However, during transient of phase current and circulating current, as shown in Fig. 15(b) and Fig. 15(c) respectively, several outliers in region II and III appear which reflect the attempt of the system to adapt to the new reference during the transient. Thus, the proposed rigorous solution should be adopted as the preferred solution for a better dynamic and safer operation.

\section{Simulation Results of AN MMC with $N=200$}

To evaluate the validity of the proposed strategy in a realscale MMC system with large number of voltage levels, a simulation test of a grid-connected MMC with 200 SMs per arm (the parameters are also summarized in Table I) is conducted in the Matlab-Plecs environment. Results are illustrated in Fig. 16 in terms of phase currents, circulating 

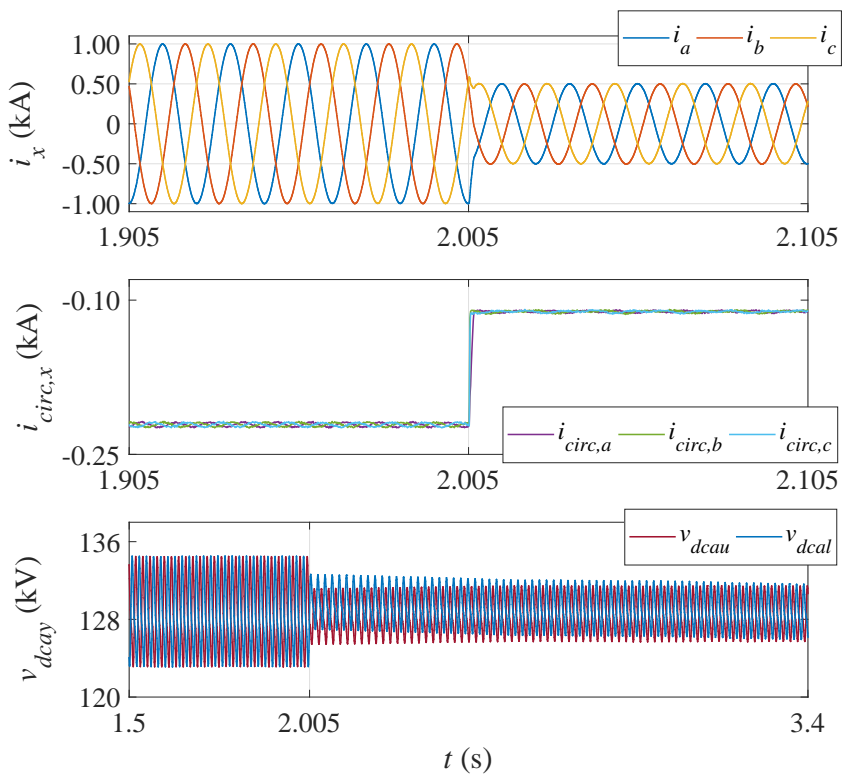

Fig. 16. Simulation results of a grid-connected MMC in a dynamic test.

currents and phase- $a$ arm energies (represented by total SMcapacitor voltage of arm). Only the rigorous solution with $J_{i w}$ $\left(\lambda_{1}=1, \lambda_{2}=25, \lambda_{3}=2 \times 10^{-8}, \lambda_{4}=1 \times 10^{-9}\right)$ is investigated since the other two methods (simplified solution and conventional MPC scheme) achieve very similar results and have been fully evaluated in the experimental section.

At first, the system operates smoothly in steady state with high-quality phase current $(\mathrm{THD}=0.18 \%)$, negligible circulating-current ripples and well-regulated arm energy. The converter works in rectifier mode with an active power of 85.5 MW and a reactive power of $0 \mathrm{VA}$. At $2.005 \mathrm{~s}$, a transient condition is generated by reducing the peak amplitude of phase-current references to half, representing a step change of active-power command. It can be observed that the system presents very high dynamics and all control objectives are well regulated in a fast transition to the new steady state (with a phase-current THD of $0.35 \%$ ). Thus, the feasibility of the proposed technique in a large-scale MMC is validated. It is worth emphasizing that even for an MMC with such high number of SMs, the proposed MPC technique still requires only 4 cost-function evaluations per phase per control cycle for the determination of arm-voltage levels.

\section{CONCLUSION}

This paper proposes a new MPC method to be applied to the MMC, which can be regarded as a hybrid method based on the FCS-MPC and CCS-MPC techniques. Utilizing the predictions based on the discrete system model, the minimization of cost function is transformed into a constrained quadratic programming problem, and the arm voltage references can be obtained analytically following the proposed rigorous routines. Considering normal operation of the MMC, a simplified procedure is provided. The upcoming modulation stage adopts the VRLC pseudomodulation plus the capacitor voltage sorting scheme.

Several experiments have been conducted on a scaleddown MMC setup. Compared with the conventional FCS-
MPC scheme that considers the entire feasible control set, the proposed method presents lower computational cost to determine the insertion-indexes. In addition, it is important to highlight that this computational cost is constant regardless of the number of SMs. Applying the proposed control methods, a very similar performance has been achieved in terms of both steady-state waveform quality and dynamic response. Further tests of circulating current transient and visualization of unconstrained solutions have revealed the significance of the proposed rigorous solutions in transient and potentially faulty conditions.

The proposed control methods open the possibility to use MPC methods for MMC with large number of SMs overcoming the issue of the high computational cost of these techniques.

\section{APPENDIX A}

The rigorous solutions of (32)/(38) are provided in the Table III, where the corresponding subscript of $i / i w$ will be added to the related variables depending on the applied cost function.

\section{REFERENCES}

[1] A. Lesnicar and R. Marquardt, "An innovative modular multilevel converter topology suitable for a wide power range," in Power Tech Conference Proceedings, 2003 IEEE Bologna, vol. 3. IEEE, 2003, pp. 6-pp.

[2] M. A. Perez, S. Bernet, J. Rodriguez, S. Kouro, and R. Lizana, "Circuit topologies, modeling, control schemes, and applications of modular multilevel converters," IEEE transactions on power electronics, vol. 30, no. 1, pp. 4-17, 2014.

[3] A. Dekka, B. Wu, R. L. Fuentes, M. Perez, and N. R. Zargari, "Evolution of topologies, modeling, control schemes, and applications of modular multilevel converters," IEEE Journal of Emerging and Selected Topics in Power Electronics, vol. 5, no. 4, pp. 1631-1656, 2017.

[4] K. Friedrich, "Modern hvdc plus application of vsc in modular multilevel converter topology," in Industrial Electronics (ISIE), 2010 IEEE International Symposium on. IEEE, 2010, pp. 3807-3810.

[5] M. Espinoza-B, R. Cárdenas, J. Clare, D. Soto-Sanchez, M. Diaz, E. Espina, and C. M. Hackl, "An integrated converter and machine control system for mmc-based high-power drives," IEEE Transactions on Industrial Electronics, vol. 66, no. 3, pp. 2343-2354, 2018.

[6] X. He, J. Peng, P. Han, Z. Liu, S. Gao, and P. Wang, "A novel advanced traction power supply system based on modular multilevel converter," IEEE Access, vol. 7, pp. 165 018-165 028, 2019.

[7] A. Antonopoulos, L. Angquist, and H.-P. Nee, "On dynamics and voltage control of the modular multilevel converter," in Power Electronics and Applications, 2009. EPE'09. 13th European Conference on. IEEE, 2009, pp. 1-10.

[8] K. Ilves, L. Harnefors, S. Norrga, and H.-P. Nee, "Analysis and operation of modular multilevel converters with phase-shifted carrier pwm," IEEE Transactions on Power Electronics, vol. 30, no. 1, pp. 268-283, 2015.

[9] B. Li, R. Yang, D. Xu, G. Wang, W. Wang, and D. Xu, "Analysis of the phase-shifted carrier modulation for modular multilevel converters," IEEE Transactions on Power Electronics, vol. 30, no. 1, pp. 297-310, 2015.

[10] J. I. Leon, S. Kouro, L. G. Franquelo, J. Rodriguez, and B. Wu, "The essential role and the continuous evolution of modulation techniques for voltage-source inverters in the past, present, and future power electronics," IEEE Transactions on Industrial Electronics, vol. 63, no. 5, pp. 2688-2701, 2016

[11] L. Angquist, A. Antonopoulos, D. Siemaszko, K. Ilves, M. Vasiladiotis, and H.-P. Nee, "Open-loop control of modular multilevel converters using estimation of stored energy," IEEE transactions on industry applications, vol. 47, no. 6, pp. 2516-2524, 2011.

[12] P. M. Meshram and V. B. Borghate, "A simplified nearest level control (nlc) voltage balancing method for modular multilevel converter (mmc)," IEEE Transactions on Power Electronics, vol. 30, no. 1, pp. 450-462, 2015. 
TABLE III

PROPOSED RIGOROUS SOLUTIONS OF (32)/(38).

\begin{tabular}{|c|c|c|c|c|}
\hline $\begin{array}{c}\text { Region of } \\
\left(v_{x u_{-} 0}, v_{x l_{-} 0}\right)\end{array}$ & Coordinates of $E_{1}, E_{2}$ & Case & If & Solution \\
\hline $\mathrm{I}$ & - & - & - & $\left(v_{x u \_0}, v_{x l \_0}\right)$ \\
\hline \multirow{3}{*}{ II-1 } & \multirow{3}{*}{$\mathrm{E}_{1}\left(-\frac{q_{2} v_{d c x l}+c_{1}}{q_{1}}, v_{d c x l}\right)$} & 1 & $0 \leq v_{x u_{-} E 1} \leq v_{d c x u}$ & $\left(v_{x u_{-} E 1}, v_{d c x l}\right)$ \\
\hline & & 2 & $v_{x u_{-} E 1}<0$ & $\left(0, v_{d c x l}\right)$ \\
\hline & & 3 & $v_{x u_{-} E 1}>v_{d c x u}$ & $\left(v_{d c x u}, v_{d c x l}\right)$ \\
\hline \multirow{3}{*}{ II- 2} & \multirow{3}{*}{$\mathrm{E}_{2}\left(0,-\frac{c_{2}}{q_{3}}\right)$} & 1 & $0 \leq v_{x l_{-} E 2} \leq v_{d c x l}$ & $\left(0, v_{x l} E 2\right)$ \\
\hline & & 2 & $v_{x l \_E 2}<0$ & $(0,0)$ \\
\hline & & 3 & $v_{x l \_E 2}>v_{d c x l}$ & $\left(0, v_{d c x l}\right)$ \\
\hline \multirow{3}{*}{ II-3 } & \multirow{3}{*}{$\mathrm{E}_{1}\left(-\frac{c_{1}}{q_{1}}, 0\right)$} & 1 & $0 \leq v_{x u_{-} E 1} \leq v_{d c x u}$ & $\left(v_{x u_{-} E 1}, 0\right)$ \\
\hline & & 2 & $v_{x u \_E 1}<0$ & $(0,0)$ \\
\hline & & 3 & $v_{x u_{-} E 1}>v_{d c x u}$ & $\left(v_{d c x u}, 0\right)$ \\
\hline \multirow{3}{*}{ II-4 } & \multirow{3}{*}{$\mathrm{E}_{2}\left(v_{d c x u},-\frac{q_{2} v_{d c x u}+c_{2}}{q_{3}}\right)$} & 1 & $0 \leq v_{x l_{-} E 2} \leq v_{d c x l}$ & $\left(v_{d c x u}, v_{x l \_E 2}\right)$ \\
\hline & & 2 & $v_{x l_{-} E 2}<0$ & $\left(v_{d c x u}, 0\right)$ \\
\hline & & 3 & $v_{x l \_E 2}>v_{d c x l}$ & $\left(v_{d c x u}, v_{d c x l}\right)$ \\
\hline \multirow{5}{*}{ III-1 } & \multirow{5}{*}{$\begin{array}{c}\mathrm{E}_{1}\left(-\frac{q_{2} v_{d c x l}+c_{1}}{q_{1}}, v_{d c x l}\right) \\
\mathrm{E}_{2}\left(0,-\frac{c_{2}}{q_{3}}\right)\end{array}$} & 1 & $\begin{array}{c}v_{x u_{-} E 1}<0 \\
v_{x l_{-} E 2}>v_{d c x l}\end{array}$ & $\left(0, v_{d c x l}\right)$ \\
\hline & & 2 & $0 \leq v_{x u_{-} E 1} \leq v_{d c x u}$ & $\left(v_{x u_{-} E 1}, v_{d c x l}\right)$ \\
\hline & & 3 & $0 \leq v_{x l_{-} E 2} \leq v_{d c x l}$ & $\left(0, v_{x l \_E 2}\right)$ \\
\hline & & 4 & $v_{x u_{-} E 1}>v_{d c x u}$ & $\left(v_{d c x u}, v_{d c x l}\right)$ \\
\hline & & 5 & $v_{x l_{-} E 2}<0$ & $(0,0)$ \\
\hline \multirow{5}{*}{ III-2 } & \multirow{5}{*}{$\begin{array}{l}\mathrm{E}_{1}\left(-\frac{c_{1}}{q_{1}}, 0\right) \\
\mathrm{E}_{2}\left(0,-\frac{c_{2}}{q_{3}}\right)\end{array}$} & 1 & $\begin{array}{l}v_{x u_{-} E 1}<0 \\
v_{x l_{-} E 2}<0\end{array}$ & $(0,0)$ \\
\hline & & 2 & $0 \leq v_{x u_{-} E 1} \leq v_{d c x u}$ & $\left(v_{x u_{-} E 1}, 0\right)$ \\
\hline & & 3 & $0 \leq v_{x l_{-} E 2} \leq v_{d c x l}$ & $\left(0, v_{x l \_E 2}\right)$ \\
\hline & & 4 & $v_{x u_{-} E 1}>v_{d c x u}$ & $\left(v_{d c x u}, 0\right)$ \\
\hline & & 5 & $v_{x l_{-} E 2}>v_{d c x l}$ & $\left(0, v_{d c x l}\right)$ \\
\hline \multirow{5}{*}{ III-3 } & \multirow{5}{*}{$\begin{array}{c}\mathrm{E}_{1}\left(-\frac{c_{1}}{q_{1}}, 0\right) \\
\mathrm{E}_{2}\left(v_{d c x u},-\frac{q_{2} v_{d c x u}+c_{2}}{q_{3}}\right)\end{array}$} & 1 & $\begin{array}{c}v_{x u_{-} E 1}>v_{d c x u} \\
v_{x l_{-} E 2}<0\end{array}$ & $\left(v_{d c x u}, 0\right)$ \\
\hline & & 2 & $0 \leq v_{x u_{-} E 1} \leq v_{d c x u}$ & $\left(v_{x u \_E 1}, 0\right)$ \\
\hline & & 3 & $0 \leq v_{x l_{-} E 2} \leq v_{d c x l}$ & $\left(v_{d c x u}, v_{x l-E 2}\right)$ \\
\hline & & 4 & $v_{x u \_E 1}<0$ & $(0,0)$ \\
\hline & & 5 & $v_{x l_{-} E 2}>v_{d c x l}$ & $\left(v_{d c x u}, v_{d c x l}\right)$ \\
\hline \multirow{5}{*}{ III-4 } & \multirow{5}{*}{$\begin{array}{c}\mathrm{E}_{1}\left(-\frac{q_{2} v_{d c x l}+c_{1}}{q_{1}}, v_{d c x l}\right) \\
\mathrm{E}_{2}\left(v_{d c x u},-\frac{q_{2} v_{d c x u}+c_{2}}{q_{3}}\right)\end{array}$} & 1 & $\begin{array}{l}v_{x u_{-} E 1}>V_{d c} \\
v_{x l_{-} E 2}>V_{d c}\end{array}$ & $\left(v_{d c x u}, v_{d c x l}\right)$ \\
\hline & & 2 & $0 \leq v_{x u_{-} E 1} \leq v_{d c x u}$ & $\left(v_{x u_{-} E 1}, v_{d c x l}\right)$ \\
\hline & & 3 & $0 \leq v_{x l_{-} E 2} \leq v_{d c x l}$ & $\left(v_{d c x u}, v_{x l_{-} E 2}\right)$ \\
\hline & & 4 & $v_{x u \_E 1}<0$ & $\left(0, v_{d c x l}\right)$ \\
\hline & & 5 & $v_{x l_{-} E 2}<0$ & $\left(v_{d c x u}, 0\right)$ \\
\hline
\end{tabular}

[13] L. Lin, Y. Lin, Z. He, Y. Chen, J. Hu, and W. Li, "Improved nearest-level modulation for a modular multilevel converter with a lower submodule number," IEEE Transactions on Power Electronics, vol. 31, no. 8, pp. $5369-5377,2016$.

[14] J.-W. Moon, J.-S. Gwon, J.-W. Park, D.-W. Kang, and J.-M. Kim, "Model predictive control with a reduced number of considered states in a modular multilevel converter for hvdc system," IEEE Transactions on Power Delivery, vol. 30, no. 2, pp. 608-617, 2015.

[15] M. Vatani, B. Bahrani, M. Saeedifard, and M. Hovd, "Indirect finite control set model predictive control of modular multilevel converters," IEEE Transactions on Smart Grid, vol. 6, no. 3, pp. 1520-1529, 2015.

[16] F. Zhang, W. Li, and G. Joós, "A voltage-level-based model predictive control of modular multilevel converter," IEEE Transactions on Industrial Electronics, vol. 63, no. 8, pp. 5301-5312, 2016.

[17] P. Guo, Z. He, Y. Yue, Q. Xu, X. Huang, Y. Chen, and A. Luo, "A novel two-stage model predictive control for modular multilevel converter with reduced computation," IEEE Transactions on Industrial Electronics, vol. 66, no. 3, pp. 2410-2422, 2018.

[18] B. Gutierrez and S.-S. Kwak, "Modular multilevel converters (mmcs) controlled by model predictive control with reduced calculation burden," IEEE Transactions on Power Electronics, vol. 33, no. 11, pp. 9176-9187, 2018.

[19] X. Liu, L. Qiu, Y. Fang, Z. Peng, and D. Wang, "Finite-level-state model predictive control for sensorless three-phase four-arm modular multilevel converter," IEEE Transactions on Power Electronics, vol. 35, no. 5, pp. 4462-4466, 2019.

[20] J. I. Leon, S. Vazquez, and L. G. Franquelo, "Multilevel converters: Control and modulation techniques for their operation and industrial applications," Proceedings of the IEEE, vol. 105, no. 11, pp. 2066-2081, 2017.

[21] S. Vazquez, C. Montero, C. Bordons, and L. G. Franquelo, "Design and experimental validation of a model predictive control strategy for a vsi with long prediction horizon," in Industrial Electronics Society, IECON 2013-39th Annual Conference of the IEEE. IEEE, 2013, pp. $5788-5793$.

[22] S. Mariéthoz and M. Morari, "Explicit model-predictive control of a pwm inverter with an lcl filter," IEEE Transactions on Industrial Electronics, vol. 56, no. 2, pp. 389-399, 2009. 
[23] H. Mahmoudi, M. Aleenejad, and R. Ahmadi, "Modulated model predictive control of modular multilevel converters in vsc-hvdc systems," IEEE Transactions on Power Delivery, vol. 33, no. 5, pp. 2115-2124, 2017.

[24] A. Mora, M. Urrutia, R. Cárdenas, A. Angulo, M. Espinoza, M. Diaz, and P. Lezana, "Model-predictive-control-based capacitor voltage balancing strategies for modular multilevel converters," IEEE Transactions on Industrial Electronics, vol. 66, no. 3, pp. 2432-2443, 2018.

[25] J. Wang, X. Liu, Q. Xiao, D. Zhou, H. Qiu, and Y. Tang, "Modulated model predictive control for modular multilevel converters with easy implementation and enhanced steady-state performance," IEEE Transactions on Power Electronics, 2020.

[26] J. Yin, A. Marquez, J. I. Leon, L. G. Franquelo, and S. Vazquez, "Improving the operation of the modular multilevel converters with model predictive control," in Compatibility, Power Electronics and Power Engineering (CPE-POWERENG), 2018 IEEE 12th International Conference on. IEEE, 2018, pp. 1-6.

[27] J. Nocedal and S. J. Wright, Sequential quadratic programming. Springer, 2006.

[28] J. L. Jerez, P. J. Goulart, S. Richter, G. A. Constantinides, E. C. Kerrigan, and M. Morari, "Embedded online optimization for model predictive control at megahertz rates," IEEE Transactions on Automatic Control, vol. 59, no. 12, pp. 3238-3251, 2014.

[29] V. Arredondo, M. A. Perez, and J. R. Espinoza, "Capacitor voltage ripple control based on decoupled power analysis in mmc," in 2017 11th IEEE International Conference on Compatibility, Power Electronics and Power Engineering (CPE-POWERENG). IEEE, 2017, pp. 544-549.

[30] P. Cortes, J. Rodriguez, C. Silva, and A. Flores, "Delay compensation in model predictive current control of a three-phase inverter," IEEE Transactions on Industrial Electronics, vol. 59, no. 2, pp. 1323-1325, 2012.

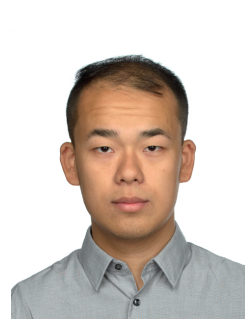

Jiapeng Yin (S'17) was born in Shenyang, China He received the B.S. degree in automation in 2014 and the M.S. degree in control science and engineering in 2016, both from the Harbin Institute of Technology, Harbin, China. He is currently working toward the Ph.D. degree with the Universidad de Sevilla, Seville, Spain. His research interests include modulation and control of cascaded multilevel converters.

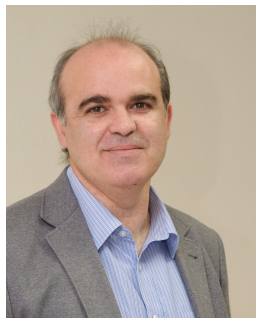

Jose I. Leon (S'04-M'07-SM'14-F'17) was born in Cadiz, Spain. He received the B.S., M.S., and $\mathrm{PhD}$ degrees in telecommunications engineering from $\mathrm{U}$ niversidad de Sevilla (US), Seville, Spain, in 1999, 2001, and 2006, respectively.

Currently, he is an Associate Professor with the Department of Electronic Engineering, US. Since 2019, he is also the Chair Professor with the Department of Control Science and Engineering in Harbin Institute of Technology, Harbin, China. His research interests include modulation and control of power converters for high-power applications and renewable energy systems.

Dr. Leon was a co-recipient of the 2008 Best Paper Award of IEEE Industrial Electronics Magazine, the 2012 Best Paper Award of the IEEE Transactions on Industrial Electronics, and the 2015 Best Paper Award of IEEE Industrial Electronics Magazine. He was the recipient of the 2014 IEEE J. David Irwin Industrial Electronics Society Early Career Award, the 2017 IEEE Bimal K. Bose Energy Systems Award and the 2017 Manuel Losada Villasante Award for excellence in research and innovation. In 2017 he was elevated to the IEEE fellow grade with the following citation "for contributions to high-power electronic converters".

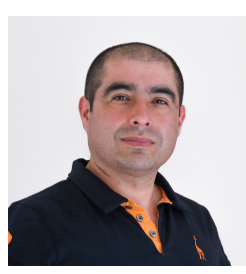

Marcelo A. Perez (M'07-SM'14) was born in Concepcion, Chile, in 1976. He received the M.Sc. and D.Sc. degree in electrical engineering from the University of Concepcion, Concepcion, Chile, in 2003 and 2006, respectively.

From 2006 to 2009, he held a postdoctoral position, and from 2009 to 2013, he worked as an associate researcher at the Universidad Tecnica Federico Santa Maria, Valparaiso, Chile. From 2013 to 2015, Dr. Perez worked as a research fellow at the Technical University of Dresden, Dresden, Germany. In 2015 he became an assistant professor at the department of electronic engineering at Universidad Tecnica Federico Santa Maria, and since 2019 he is an associate professor at the same institution.

Dr. Perez is a principal researcher at the Advanced Center for Electric and Electronic Engineering (AC3E) and associated researcher at the Solar Energy Research Center (SERC- Chile). He has co-authored two book chapters and more than 130 journal and conference papers. Dr. Perez was the recipient of the IEEE Industry Applications Magazine 1st prize paper award of 2012, the second-best paper award in the IEEE Transactions on Power Electronics in 2016. He was the IEEE-IES Region 9 Chapter Coordinator, and currently, he is President of the IEEE-IES Chapter Chile. Dr. Perez is Associate Editor in the IEEE Transactions on Power Electronics and the IEEE Transactions on Industrial Electronics.

His main interests are multilevel power converters topologies and its control for applications in electromobility, smart grids, HVDC systems, and energy harvesting.

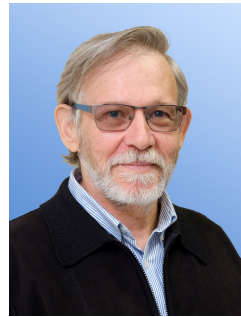

Leopoldo G. Franquelo (M'84-SM'96-F'05) was born in Málaga, Spain. He received the M.Sc. and $\mathrm{Ph} . \mathrm{D}$. degrees in electrical engineering from the Universidad de Sevilla, Seville, Spain, in 1977 and 1980 , respectively. He was associate professor from 1982-86 at the Electronics Engineering Department in Sevilla University and currently is professor at the Electronics Engineering Department in Sevilla University since 1986 and a 1000 Talent Professor at the Department of Control Science and Engineering in Harbin Institute of Technology since 2016 His current research interests include modulation techniques for multilevel inverters and application to power electronic systems for renewable energy systems. He has participated in more than 100 Industrial and R\&D projects and has published more than 300 papers, 76 of them in IEEE Journals.

Dr. Franquelo is an IEEE Fellow since 2005 and an IEEE Industrial Electronics Society (IES) Distinguished Lecturer since 2006. In the IEEE TRANSACTIONS ON INDUSTRIAL ELECTRONICS, he became an Associate Editor in 2007, Co-Editor-in-Chief in 2014, and the Editor-in-Chief since 2016. He was a Member-at-Large of the IES AdCom (2002-2003), Vice President for Conferences (2004-2007), and President Elect of the IES (20082009). He was the President of the IES (2010-2011) and is an IES AdCom Life member. In 2009 and 2013, he received the prestigious Andalusian Research Award and FAMA Award recognizing the excellence of his research career. $\mathrm{He}$ has received a number of Best Paper Awards from IEEE journals. In 2012 and 2015, he was the recipient of the Eugene Mittelmann Outstanding Research Achievement Award and the Antohny J. Hornfeck Service Award from IEEE-IES, respectively.

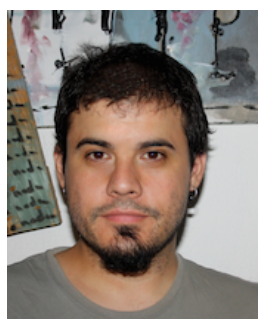

Abraham Marquez (S'14-M'16) was born in Huelva, Spain, in 1985. He received his B.S., M.S. and $\mathrm{Ph} . \mathrm{D}$ degrees in telecommunications engineering from Universidad de Sevilla (US), Seville, Spain in 2014, 2016 and 2019, respectively. His main research interest are modulation techniques, multilevel converters, model-based predictive control of power converters and drives, renewable energy sources, and power devices lifetime extension. Dr. Marquez was recipient as coauthor of the 2015 Best Paper Award of the IEEE Industrial Electronics Magazine. 


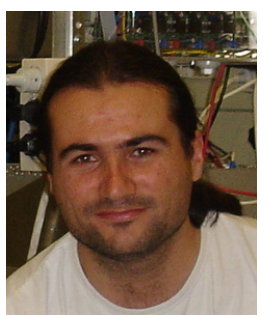

Sergio Vazquez (S'04-M'08-SM'14) was born in Seville, Spain, in 1974. He received the M.S. and $\mathrm{PhD}$ degrees in industrial engineering from the University of Seville (US) in 2006, and 2010, respectively.

Since 2002, he is with the Power Electronics Group working in $\mathrm{R} \& \mathrm{D}$ projects. $\mathrm{He}$ is an Associate Professor with the Department of Electronic Engineering, US. His research interests include power electronics systems, modeling, modulation and control of power electronics converters applied to renewable energy technologies.

Dr. Vazquez was recipient as coauthor of the 2012 Best Paper Award of the IEEE Transactions on Industrial Electronics and 2015 Best Paper Award of the IEEE Industrial Electronics Magazine. He is involved in the Energy Storage Technical Committee of the IEEE industrial electronics society and is currently serving as an Associate Editor of the IEEE Transactions on Industrial Electronics. 